\title{
Properties-preserving high order numerical methods for a kinetic eikonal equation
}

\author{
Songting Luo ${ }^{\mathrm{a}, *}$, Nicholas Payne ${ }^{\mathrm{a}}$ \\ ${ }^{a}$ Department of Mathematics, Iowa State University, Ames, IA 50011.
}

\begin{abstract}
For the BGK (Bhatnagar-Gross-Krook) equation in the large scale hyperbolic limit, the density of particles can be transformed as the Hopf-Cole transformation, where the phase function converges uniformly to the viscosity solution of an effective Hamilton-Jacobi equation, referred to as the kinetic eikonal equation. In this work, we present efficient high order finite difference methods for numerically solving the kinetic eikonal equation. The methods are based on monotone schemes such as the Godunov scheme. High order weighted essentially non-oscillatory techniques and Runge-Kutta procedures are used to obtain high order accuracy in both space and time. The effective Hamiltonian is determined implicitly by a nonlinear equation given as integrals with respect to the velocity variable. Newton's method is applied to solve the nonlinear equation, where integrals with respect to the velocity variable are evaluated either by a Gauss quadrature formula or as expansions with respect to moments of the Maxwellian. The methods are designed such that several key properties such as the positivity of the viscosity solution and the positivity of the effective Hamiltonian are preserved. Numerical experiments are presented to demonstrate the effectiveness of the methods.
\end{abstract}

Keywords: BGK equation, Hopf-Cole transformation, kinetic eikonal equation, WENO, Runge-Kutta, Godunov, properties-preserving

2010 MSC: 65M06, 35Q20, 35Q83, 35F21

\section{Introduction}

We consider the kinetic equation with BGK (Bhatnagar-Gross-Krook) relaxation operator in the large scale hyperbolic limit [2],

$$
\partial_{t} f^{\epsilon}+\mathbf{v} \cdot \nabla_{\mathbf{x}} f^{\epsilon}=\frac{1}{\epsilon}\left(M(\mathbf{v}) \rho^{\epsilon}-f^{\epsilon}\right),(t, \mathbf{x}, \mathbf{v}) \in \mathbb{R}_{+} \times \mathbb{R}^{n} \times \mathbb{V}
$$

\footnotetext{
This work is partially supported by NSF DMS 1418908 .

${ }^{*}$ Corresponding author

Email addresses: luos@iastate.edu (Songting Luo), napayne@iastate.edu (Nicholas Payne)
} 
where $f^{\epsilon}(t, \mathbf{x}, \mathbf{v})$ denotes the density of particles moving with speed $\mathbf{v} \in \mathbb{V}$ at time $t \in \mathbb{R}_{+}$and position $\mathbf{x} \in$ $\mathbb{R}^{n}, \mathbb{V} \subset \mathbb{R}^{n}$ is bounded and symmetric, $\epsilon$ represents the Knudsen number, $\rho^{\epsilon}(t, \mathbf{x})$ denotes the macroscopic density of particles, i.e.,

$$
\rho^{\epsilon}(t, \mathbf{x})=\int_{\mathbb{V}} f^{\epsilon}(t, \mathbf{x}, \mathbf{v}) d \mathbf{v},(t, \mathbf{x}) \in \mathbb{R}_{+} \times \mathbb{R}^{n},
$$

and $M$ is the Maxwellian which is symmetric and satisfies the following moment identities:

$$
\int_{\mathbb{V}} M(\mathbf{v}) d \mathbf{v}=1, \int_{\mathbb{V}} \mathbf{v} M(\mathbf{v}) d \mathbf{v}=\mathbf{0}, \int_{\mathbb{V}} \mathbf{v}^{2} M(\mathbf{v}) d \mathbf{v}=\theta^{2}
$$

As $\epsilon \rightarrow 0$, the velocity distribution relaxes rapidly towards the Maxwellian distribution, which motivates the following Hopf-Cole transformation of the density:

$$
f^{\epsilon}(t, \mathbf{x}, \mathbf{v})=M(\mathbf{v}) e^{-\frac{\psi^{\epsilon}(t, \mathbf{x}, \mathbf{v})}{\epsilon}},
$$

where $\psi^{\epsilon}$ is the phase function and has several uniform estimates with respect to $\epsilon>0$ [3]:

Proposition 1.1. Let $\mathbb{V} \subset \mathbb{R}^{n}$ be a bounded subset. Assume $M \in L^{1}(\mathbb{V})$, and is nonnegative and symmetric, $\psi^{\epsilon}(0, \boldsymbol{x}, \boldsymbol{v})=\psi_{0}(\boldsymbol{x}) \geq 0$, and $\psi_{0} \in W^{1, \infty}\left(\mathbb{R}^{n}\right)$, then

$$
\begin{aligned}
& 0 \leq \psi^{\epsilon}(t, \cdot, \cdot) \leq\left\|\psi_{0}\right\|_{\infty} \\
& \left\|\nabla_{x} \psi^{\epsilon}(t, \cdot, \cdot)\right\|_{\infty} \leq\left\|\nabla_{x} \psi_{0}\right\|_{\infty}, \\
& \left\|\nabla_{\boldsymbol{v}} \psi^{\epsilon}(t, \cdot, \cdot)\right\|_{\infty} \leq t\left\|\nabla_{x} \psi_{0}\right\|_{\infty} \\
& \left\|\partial_{t} \psi^{\epsilon}(t, \cdot, \cdot)\right\|_{\infty} \leq V_{\max }\left\|\nabla_{x} \psi_{0}\right\|_{\infty},
\end{aligned}
$$

where $V_{\max }$ is the maximum speed.

It has been proved that $\psi^{\epsilon}(t, \mathbf{x}, \mathbf{v}) \rightarrow \psi(t, \mathbf{x})$ (locally) uniformly as $\epsilon \rightarrow 0$. The limit function $\psi$ is independent of $\mathbf{v}$ and is the viscosity solution of an effective Hamilton-Jacobi equation [3].

Theorem 1.1. Let $\mathbb{V} \subset \mathbb{R}^{n}$ be bounded and symmetric, and $M \in L^{1}(\mathbb{V})$ be nonnegative and symmetric. Then $\psi^{\epsilon}$ converges (locally) uniformly towards $\psi$, where $\psi$ is the viscosity solution of the following effective Hamilton-Jacobi equation (referred to as the kinetic eikonal equation):

$$
\int_{\mathbb{V}} \frac{M(\boldsymbol{v})}{1-\partial_{t} \psi(t, \boldsymbol{x})-\boldsymbol{v} \cdot \nabla_{x} \psi(t, \boldsymbol{x})} d \boldsymbol{v}=1,(t, \boldsymbol{x}) \in \mathbb{R}_{+} \times \mathbb{R}^{n}
$$

The denominator of the integrand is positive for all $\boldsymbol{v} \in \mathbb{V}$. 
The kinetic eikonal equation (7) can be recast as

$$
\partial_{t} \psi(t, \mathbf{x})+H\left(\nabla_{\mathbf{x}} \psi\right)=0
$$

with the effective Hamiltonian $H(\mathbf{p})$ defined implicitly by

$$
\int_{\mathbb{V}} \frac{M(\mathbf{v})}{1+H(\mathbf{p})-\mathbf{v} \cdot \mathbf{p}} d \mathbf{v}=1
$$

where $\mathbf{p}=\nabla_{\mathbf{x}} \psi(t, \mathbf{x})[3]$.

In this work, we design efficient high order finite difference methods for solving the kinetic eikonal equation (8) numerically. This work provides necessary components that are important in a forthcoming work that

The BGK equation is a simpler relaxation model associated with the Boltzmann equation for the kinetic description of gases. The importance of the BGK model in applications has motivated the development of many numerical methods tailored to particular structures of BGK equations; see for instance [16, 17, 11] and references therein. Our ultimate goal is to design an effective method for the BGK equation (8) that

3. $\left\|\nabla_{\mathbf{p}} H\right\|_{\infty} \leq V_{\max }$, where $V_{\max }$ is the maximum speed, which can be derived by differentiating equation (9) with respect to $\mathbf{p}[3$, i.e.,

$$
\int_{\mathbb{V}} \frac{M(\mathbf{v})}{(1+H(\mathbf{p})-\mathbf{v} \cdot \mathbf{p})^{2}}\left(\nabla_{\mathbf{p}} H(\mathbf{p})-\mathbf{v}\right) d \mathbf{v}=0
$$

4. $H(\mathbf{p})$ is convex, which can be derived by differentiating equation $(9)$ with respect to $\mathbf{p}$ twice [3] , i.e.,

$$
\left(\int_{\mathbb{V}} \frac{M(\mathbf{v})}{(1+H(\mathbf{p})-\mathbf{v} \cdot \mathbf{p})^{2}} d \mathbf{v}\right) D_{\mathbf{p}}^{2} H(\mathbf{p})=2 \int_{\mathbb{V}} \frac{M(\mathbf{v})}{(1+H(\mathbf{p})-\mathbf{v} \cdot \mathbf{p})^{3}}\left(\nabla_{\mathbf{p}} H(\mathbf{p})-\mathbf{v}\right) \otimes\left(\nabla_{\mathbf{p}} H(\mathbf{p})-\mathbf{v}\right) d \mathbf{v} .
$$

5. $H(\mathbf{0})=0$ and $\nabla_{\mathbf{p}} H(\mathbf{0})=\mathbf{0}$, which can be derived from equation 9 and equation 10 , respectively, 
i.e.,

$$
\int_{\mathbb{V}} \frac{M(\mathbf{v})}{1+H(\mathbf{0})} d \mathbf{v}=1 \Rightarrow H(\mathbf{0})=0
$$

and

$$
\int_{\mathbb{V}} \frac{M(\mathbf{v})}{(1+H(\mathbf{0}))^{2}}\left(\nabla_{\mathbf{p}} H(\mathbf{0})-\mathbf{v}\right) d \mathbf{v}=0 \Rightarrow \nabla_{\mathbf{p}} H(\mathbf{0})=\mathbf{0} .
$$

6. $H(\mathbf{p}) \geq 0$, and $H(\mathbf{p})=0$ only if $\mathbf{p}=\mathbf{0}$, which is obvious from the above observations, i.e., convexity of $H(\mathbf{p})$.

For computing $\psi$ numerically by solving the kinetic eikonal equation $(8)$, with the effective Hamiltonian $H(\mathbf{p})$ determined as a root of the nonlinear equation (9), there are two major obstacles: (1) the above properties and has high order accuracy both in space and time.

The rest of the paper is organized as follows. In Section 2, we present the numerical schemes proposed to solve the kinetic eikonal equation. In Section 3, we present numerical experiments to demonstrate our methods. Concluding remarks along with discussion of future work are given in Section 4

\section{Properties-Preserving Schemes}

We present the numerical methods for solving the kinetic eikonal equation (8) with equation (9). For simplicity of exposition, we present the schemes in 2-D $(n=2)$, and denote $\mathbf{x}=(x, y), \mathbf{v}=(u, v)$, and $\nabla_{\mathbf{x}} \psi=\left(\psi_{x}, \psi_{y}\right)$. Assume the computational domain is $\Omega \equiv\left\{(x, y): x_{\min } \leq x \leq x_{\max }, y_{\min } \leq y \leq y_{\max }\right\}$, where periodic boundary conditions in $(x, y)$ are imposed, and the velocity domain is $\mathbb{V} \equiv\left\{(u, v): u_{\min } \leq\right.$ $\left.u \leq u_{\max }, v_{\min } \leq v \leq v_{\max }\right\}$. The domain $\Omega$ is discretized and covered by a uniform mesh $\Omega^{h} \equiv\left\{\left(x_{i}, y_{j}\right)\right.$ : $\left.x_{i}=x_{\min }+i \Delta x, y_{j}=y_{\min }+j \Delta y ; 0 \leq i \leq N_{x}, 0 \leq j \leq N_{y}\right\}$ with mesh sizes $\Delta x=\left(x_{\max }-x_{\min }\right) / N_{x}$, 
and $\Delta y=\left(y_{\max }-y_{\min }\right) / N_{y}$. And the time step size is denoted as $\Delta t$. For mesh sizes $\Delta x, \Delta y, \Delta t, \psi_{i j}^{k}$ will denote a numerical approximation to the viscosity solution $\psi\left(t^{k}, x_{i}, y_{j}\right)=\psi\left(k \Delta t, x_{\min }+i \Delta x, y_{\min }+j \Delta y\right)$. Some standard notations will be used such as

$$
\Delta_{ \pm}^{x} \psi_{i j}= \pm\left(\psi_{i \pm 1, j}-\psi_{i j}\right), \text { and } \Delta_{ \pm}^{y} \psi_{i j}= \pm\left(\psi_{i, j \pm 1}-\psi_{i j}\right)
$$

\subsection{Numerical Hamiltonian}

The starting point is the first order monotone scheme [5],

$$
\psi_{i j}^{k+1}=\psi_{i j}^{k}-\Delta t \hat{H}\left(\frac{\Delta_{+}^{x} \psi_{i j}^{k}}{\Delta x}, \frac{\Delta_{-}^{x} \psi_{i j}^{k}}{\Delta x}, \frac{\Delta_{+}^{y} \psi_{i j}^{k}}{\Delta y}, \frac{\Delta_{-}^{y} \psi_{i j}^{k}}{\Delta y}\right)
$$

where $\hat{H}$ is a monotone numerical Hamiltonian consistent with $H$, i.e.,

$$
\hat{H}(p, p, q, q)=H(p, q)
$$

$\hat{H}$ is non-increasing in its first and third arguments and nondecreasing in the other two, and the time step size $\Delta t$ is chosen such that the CFL condition is satisfied, i.e.,

$$
V_{\max }\left(\frac{\Delta t}{\Delta x}+\frac{\Delta t}{\Delta y}\right) \leq 1
$$

It is clear that the right hand side of 111), denoted as $\hat{F}\left(\psi_{i j}^{k}, \psi_{i \pm 1, j}^{k}, \psi_{i, j \pm 1}^{k}\right)$, is nondecreasing in $\left\{\psi_{i j}^{k}, \psi_{i \pm 1, j}^{k}, \psi_{i, j \pm 1}^{k}\right\}$. Therefore, if $\psi_{\min } \leq\left\{\psi_{i j}^{k}\right\} \leq \psi_{\max }$ for some constants $\psi_{\min }$ and $\psi_{\max }$, then $\psi_{\min } \leq\left\{\psi_{i j}^{k+1}\right\} \leq \psi_{\max }$.

We will consider the following Godunov numerical Hamiltonian [1, 15]:

$$
\hat{H}^{G}\left(p^{+}, p^{-}, q^{+}, q^{-}\right)=\operatorname{ext}_{p \in I\left(p^{-}, p^{+}\right)} \operatorname{ext}_{q \in I\left(q^{-}, q^{+}\right)} H(p, q)
$$

where

$$
\operatorname{ext}_{p \in I(a, b)}=\left\{\begin{array}{l}
\min _{a \leq p \leq b} \text { if } a \leq b \\
\max _{b \leq p \leq a} \text { if } a>b .
\end{array}\right.
$$

The Godunov Hamiltonian $\hat{H}^{G}$ is monotone for $A \leq p^{ \pm} \leq B, C \leq q^{ \pm} \leq D$, with $A, B, C$ and $D$ appropriate 45 constants. In the Introduction (Section 1 we observed that $H(p, q) \geq 0$. Therefore, we want the numerical Hamiltonian $\hat{H}$ to satisfy $\hat{H}\left(p^{+}, p^{-}, q^{+}, q^{-}\right) \geq 0$. Note that the Godunov Hamiltonian $\hat{H}^{G}\left(p^{+}, p^{-}, q^{+}, q^{-}\right) \geq$ 0 as long as $H(p, q) \geq 0$ for $A \leq p \leq B, C \leq q \leq D$. 
To preserve the positivity of the Hamiltonian, we choose $\hat{H}$ to be $\hat{H}^{G}$. Now we describe the high order schemes. We use the WENO constructions to approximate $p^{ \pm}=\psi_{x}^{ \pm}, q^{ \pm}=\psi_{y}^{ \pm}$, and the Runge-Kutta procedures to update the solution in time.

\subsection{WENO Constructions and Runge-Kutta Procedures}

The WENO constructions for approximating $\psi_{x}^{ \pm}$and $\psi_{y}^{ \pm}$are given as the following. For third order WENO construction (WENO3) [13, 10, 18, we have

$$
\left(\psi_{x}^{-}\right)_{i j}=\left(1-\omega_{-}\right) \frac{\psi_{i+1, j}-\psi_{i-1, j}}{2 \Delta x}+\omega_{-} \frac{3 \psi_{i j}-4 \psi_{i-1, j}+\psi_{i-2, j}}{2 \Delta x},
$$

with

$$
\omega_{-}=\frac{1}{1+2 \gamma_{-}^{2}}, \gamma_{-}=\frac{\epsilon_{0}+\left(\psi_{i j}-2 \psi_{i-1, j}+\psi_{i-2, j}\right)^{2}}{\epsilon_{0}+\left(\psi_{i+1, j}-2 \psi_{i j}+\psi_{i-1, j}\right)^{2}}
$$

and

$$
\left(\psi_{x}^{+}\right)_{i j}=\left(1-\omega_{+}\right) \frac{\psi_{i+1, j}-\psi_{i-1, j}}{2 \Delta x}+\omega_{+} \frac{-3 \psi_{i j}+4 \psi_{i+1, j}-\psi_{i+2, j}}{2 \Delta x},
$$

with

$$
\omega_{+}=\frac{1}{1+2 \gamma_{+}^{2}}, \gamma_{+}=\frac{\epsilon_{0}+\left(\psi_{i j}-2 \psi_{i+1, j}+\psi_{i+2, j}\right)^{2}}{\epsilon_{0}+\left(\psi_{i+1, j}-2 \psi_{i j}+\psi_{i-1, j}\right)^{2}} .
$$

WENO3 approximations for $\psi_{y}^{ \pm}$can be constructed similarly. $\epsilon_{0}$ is chosen as $10^{-6}$ to avoid division by zero.

For fifth order WENO construction (WENO5) [13, 10, 18, we have

$$
\begin{aligned}
\left(\psi_{x}^{-}\right)_{i j} & =\frac{1}{12 \Delta x}\left(-\Delta_{+}^{x} \psi_{i-2, j}+7 \Delta_{+}^{x} \psi_{i-1, j}+7 \Delta_{+}^{x} \psi_{i j}-\Delta_{+}^{x} \psi_{i+1, j}\right) \\
& -\Phi^{W E N O}\left(\frac{\Delta_{-}^{x} \Delta_{+}^{x} \psi_{i-2, j}}{\Delta x}, \frac{\Delta_{-}^{x} \Delta_{+}^{x} \psi_{i-1, j}}{\Delta x}, \frac{\Delta_{-}^{x} \Delta_{+}^{x} \psi_{i j}}{\Delta x}, \frac{\Delta_{-}^{x} \Delta_{+}^{x} \psi_{i+1, j}}{\Delta x}\right)
\end{aligned}
$$

where

$$
\begin{aligned}
& \Phi^{W E N O}(a, b, c, d)=\frac{1}{3} \omega_{0}(a-2 b+c)+\frac{1}{6}\left(\omega_{2}-\frac{1}{2}\right)(b-2 c+d), \\
& \omega_{0}=\frac{\alpha_{0}}{\alpha_{0}+\alpha_{1}+\alpha_{2}}, \omega_{2}=\frac{\alpha_{2}}{\alpha_{0}+\alpha_{1}+\alpha_{2}}, \\
& \alpha_{0}=\frac{1}{\left(\epsilon_{0}+I S_{0}\right)^{2}}, \alpha_{1}=\frac{6}{\left(\epsilon_{0}+I S_{1}\right)^{2}}, \alpha_{2}=\frac{3}{\left(\epsilon_{0}+I S_{2}\right)^{2}}, \\
& I S_{0}=13(a-b)^{2}+3(a-3 b)^{2} \\
& I S_{1}=13(b-c)^{2}+3(b+c)^{2} \\
& I S_{2}=13(c-d)^{2}+3(3 c-d)^{2}
\end{aligned}
$$


and

$$
\begin{aligned}
\left(\psi_{x}^{+}\right)_{i j} & =\frac{1}{12 \Delta x}\left(-\Delta_{+}^{x} \psi_{i-2, j}+7 \Delta_{+}^{x} \psi_{i-1, j}+7 \Delta_{+}^{x} \psi_{i j}-\Delta_{+}^{x} \psi_{i+1, j}\right) \\
& -\Phi^{W E N O}\left(\frac{\Delta_{-}^{x} \Delta_{+}^{x} \psi_{i+2, j}}{\Delta x}, \frac{\Delta_{-}^{x} \Delta_{+}^{x} \psi_{i+1, j}}{\Delta x}, \frac{\Delta_{-}^{x} \Delta_{+}^{x} \psi_{i j}}{\Delta x}, \frac{\Delta_{-}^{x} \Delta_{+}^{x} \psi_{i-1, j}}{\Delta x}\right) .
\end{aligned}
$$

WENO5 approximations for $\psi_{y}^{ \pm}$can be constructed similarly. $\epsilon_{0}$ is chosen as $10^{-13}$ to avoid division by zero.

If WENO3 is used, the Strong Stability-Preserving (SSP) third-order Runge-Kutta method is used for the time discretization [7, i.e.,

$$
\begin{aligned}
K_{1} & =L\left(\psi^{k-1}\right), \\
K_{2} & =L\left(\psi^{k-1}+\Delta t K_{1}\right), \\
K_{3} & =L\left(\psi^{k-1}+\Delta t\left(\frac{1}{4} K_{1}+\frac{1}{4} K_{2}\right)\right), \\
\psi^{k} & =\psi^{k-1}+\Delta t\left(\frac{1}{6} K_{1}+\frac{1}{6} K_{2}+\frac{2}{3} K_{3}\right),
\end{aligned}
$$

where $L(\psi)=-\hat{H}\left(\psi_{x}^{ \pm}, \psi_{y}^{ \pm}\right)$. If WENO5 is used, the fifth order Runge-Kutta method by Lawson is used for the time discretization [12], i.e.,

$$
\begin{aligned}
& K_{1}=L\left(\psi^{k-1}\right), \\
& K_{2}=L\left(\psi^{k-1}+\Delta t a_{21} K_{1}\right), \\
& K_{3}=L\left(\psi^{k-1}+\Delta t\left(a_{31} K_{1}+a_{32} K_{2}\right)\right), \\
& K_{4}=L\left(\psi^{k-1}+\Delta t\left(a_{41} K_{1}+a_{42} K_{2}+a_{43} K_{3}\right)\right), \\
& K_{5}=L\left(\psi^{k-1}+\Delta t\left(a_{51} K_{1}+a_{52} K_{2}+a_{53} K_{3}+a_{54} K_{4}\right)\right), \\
& K_{6}=L\left(\psi^{k-1}+\Delta t\left(a_{61} K_{1}+a_{62} K_{2}+a_{63} K_{3}+a_{64} K_{4}+a_{65} K_{5}\right)\right), \\
& \psi^{k}=\psi^{k-1}+\Delta t\left(b_{1} K_{1}+b_{2} K_{2}+b_{3} K_{3}+b_{4} K_{4}+b_{5} K_{5}+b_{6} K_{6}\right),
\end{aligned}
$$

where

$$
\left(\begin{array}{cccccc}
a_{21} & & & & \\
a_{31} & a_{32} & & & \\
a_{41} & a_{42} & a_{43} & & \\
a_{51} & a_{52} & a_{53} & a_{54} & \\
a_{61} & a_{62} & a_{63} & a_{64} & a_{65}
\end{array}\right)=\left(\begin{array}{ccccc}
1 / 12 & & & & \\
-1 / 8 & 3 / 8 & & & \\
3 / 5 & -9 / 10 & 4 / 5 & & \\
39 / 80 & -9 / 20 & 3 / 20 & 9 / 16 & \\
-59 / 35 & 66 / 35 & 48 / 35 & -12 / 7 & 8 / 7
\end{array}\right),
$$


and

$$
\left(\begin{array}{l}
b_{1} \\
b_{2} \\
b_{3} \\
b_{4} \\
b_{5} \\
b_{6}
\end{array}\right)=\left(\begin{array}{c}
7 / 90 \\
0 \\
32 / 90 \\
12 / 90 \\
32 / 90 \\
7 / 90
\end{array}\right) .
$$

We remark that the choice of Runge-Kutta procedure is not limited to the ones described above. Any Strong Stability-Preserving (SSP) or Total Variation Diminishing (TVD) Runge-Kutta methods, if applicable, can be used. Appropriate time steps must be used to obtain consistent order of accuracy in both space and time.

\subsection{Computation of Effective Hamiltonian}

For evaluating the numerical Hamiltonian $\hat{H}$, the effective Hamiltonian $H$ must be computed. Given $p, q$, the effective Hamiltonian $H(p, q)$ is determined implicitly by equation (9) which is a nonlinear function in $H$, i.e.,

$$
F(H)=0, \quad \text { with } F(H)=\int_{v_{\min }}^{v_{\max }} \int_{u_{\min }}^{u_{\max }} \frac{M(u, v)}{1+H-u p-v q} d u d v-1 .
$$

For solving the nonlinear equation, since $H \geq 0$ and $H \geq \max _{(u, v) \in \mathbb{V}}\{u p+v q-1\}$, an obvious choice is to use the Bisection method to search for the solution on the interval $H \in\left[\max \left\{0, \max _{(u, v) \in \mathbb{V}}\{u p+v q-1\}\right\}, \infty\right)$. It is easy to see that $F(H)$ is decreasing for $H \in\left[\max \left\{0, \max _{(u, v) \in \mathbb{V}}\{u p+v q-1\}\right\}, \infty\right)$, since

$$
F^{\prime}(H)=-\int_{v_{\min }}^{v_{\max }} \int_{u_{\min }}^{u_{\max }} \frac{M(u, v)}{(1+H-u p-v q)^{2}} d u d v<0 .
$$

Therefore, the solution is unique. To accelerate the convergence, we use Newton's method. Starting with an initial guess $H_{0}$, the method generates a sequence $\left\{H_{m}\right\}$ that converges to $H$ by

$$
H_{m}=H_{m-1}-\frac{F\left(H_{m-1}\right)}{F^{\prime}\left(H_{m-1}\right)}, m \geq 1 .
$$

Both $F(H)$ and $F^{\prime}(H)$ must be evaluated as integrals with respect to the velocity variable $(u, v)$. There are

- Gauss quadrature formulas: i.e.,

$$
F(H) \approx \sum_{s} \sum_{t} \frac{w_{s}^{u} w_{t}^{v}}{1+H-u_{s} p-v_{t} q}-1, F^{\prime}(H) \approx-\sum_{s} \sum_{t} \frac{w_{s}^{u} w_{t}^{v}}{\left(1+H-u_{s} p-v_{t} q\right)^{2}},
$$


where $\left\{w_{s}^{u}, u_{s}\right\}$ are weights and abscissas of an appropriate Gauss quadrature rule on $\left[u_{\min }, u_{\max }\right]$, and $\left\{w_{t}^{v}, v_{t}\right\}$ are weights and abscissas of an appropriate Gauss quadrature rule on $\left[v_{\min }, v_{\max }\right]$.

- Expansions with respect to moments of the Maxwellian $M$ : Since $1+H>u p+v q$ for $u_{\min } \leq u \leq u_{\max }$ and $v_{\min } \leq v \leq v_{\max }$, we have $1+H>|p| u_{\max }+|q| v_{\max }$. We can rewrite $F(H)$ as

$$
\begin{aligned}
F(H) & =\frac{1}{1+H} \int_{v_{\min }}^{v_{\max }} \int_{u_{\min }}^{u_{\max }} \frac{M(u, v)}{1-\frac{u p+v q}{1+H}} d u d v-1 \\
& =\frac{1}{1+H} \int_{v_{\min }}^{v_{\max }} \int_{u_{\min }}^{u_{\max }} M(u, v) \sum_{m=0}^{\infty}\left(\frac{u p+v q}{1+H}\right)^{m} d u d v-1 \\
& =\frac{1}{1+H} \int_{v_{\min }}^{v_{\max }} \int_{u_{\min }}^{u_{\max }} M(u, v) \sum_{m=0}^{\infty} \frac{\sum_{l=0}^{m} C_{m}^{l}(u p)^{l}(v q)^{m-l}}{(1+H)^{m}} d u d v-1 \\
& =\sum_{m=0}^{\infty} \frac{1}{(1+H)^{m+1}} \sum_{l=0}^{m} C_{m}^{l} p^{l} q^{m-l} \int_{v_{\min }}^{v_{\max }} \int_{u_{\min }}^{u_{\max }} M(u, v) u^{l} v^{m-l} d u d v-1 \\
& =\sum_{m=0}^{\infty} \frac{1}{(1+H)^{m+1}} \sum_{l=0}^{m} C_{m}^{l} p^{l} q^{m-l} \theta_{l, m-l}-1,
\end{aligned}
$$

where $\left\{C_{m}^{l}\right\}$ are the binomial coefficients, and $\left\{\theta_{l, m-l}\right\}$ are moments of the Maxwellian $M$,

$$
\theta_{l, m-l}=\int_{v_{\min }}^{v_{\max }} \int_{u_{\min }}^{u_{\max }} M(u, v) u^{l} v^{m-l} d u d v
$$

Similarly, we can rewrite $F^{\prime}(H)$ as

$$
\begin{aligned}
F^{\prime}(H) & =-\frac{1}{(1+H)^{2}} \int_{v_{\min }}^{v_{\max }} \int_{u_{\min }}^{u_{\max }} \frac{M(u, v)}{\left(1-\frac{u p+v q}{1+H}\right)^{2}} d u d v-1 \\
& =-\frac{1}{(1+H)^{2}} \int_{v_{\min }}^{v_{\max }} \int_{u_{\min }}^{u_{\max }} M(u, v) \sum_{m=0}^{\infty}(m+1)\left(\frac{u p+v q}{1+H}\right)^{m} d u d v \\
& =-\frac{1}{(1+H)^{2}} \int_{v_{\min }}^{v_{\max }} \int_{u_{\min }}^{u_{\max }} M(u, v) \sum_{m=0}^{\infty}(m+1) \frac{\sum_{l=0}^{m} C_{m}^{l}(u p)^{l}(v q)^{m-l}}{(1+H)^{m}} d u d v \\
& =-\sum_{m=0}^{\infty} \frac{m+1}{(1+H)^{m+2}} \sum_{l=0}^{m} C_{m}^{l} p^{l} q^{m-l} \theta_{l, m-l} .
\end{aligned}
$$

In practice, the infinity series must be truncated as

$$
\begin{aligned}
& F(H) \approx \sum_{m=0}^{T} \frac{1}{(1+H)^{m+1}} \sum_{l=0}^{m} C_{m}^{l} p^{l} q^{m-l} \theta_{l, m-l}-1, \\
& F^{\prime}(H) \approx-\sum_{m=0}^{T} \frac{m+1}{(1+H)^{m+2}} \sum_{l=0}^{m} C_{m}^{l} p^{l} q^{m-l} \theta_{l, m-l},
\end{aligned}
$$


with $T>0$ an appropriate integer.

The number of Gauss abscissas/weights or the number of moments of the Maxwellian used in the approxi-

mations of $F(H)$ and $F^{\prime}(H)$ determines the efficiency of the overall method. It turns out a relatively small set is enough to obtain high accuracy in practice; see numerical experiments in Section 3 . We summarize Newton's method for solving the nonlinear equation (9):

Algorithm 1. Newton's method for equation (9)

1. Initial guess: set $H_{0} \geq \max \left\{0,|p| u_{\max }+|q| v_{\max }-1\right\}$, according to Property 2 in Section 1 .

2. Newton's iterations: for $m \geq 1$,

$$
H_{m}=H_{m-1}-\frac{F\left(H_{m-1}\right)}{F^{\prime}\left(H_{m-1}\right)},
$$

where $F\left(H_{m-1}\right)$ and $F^{\prime}\left(H_{m-1}\right)$ are approximated by either 15) or 16). Set

$$
H_{m}=\max \left\{H_{m}, 0,|p| u_{\max }+|q| v_{\max }-1\right\} .
$$

3. Termination: if $\left|F\left(H_{m}\right)\right|<\delta$ for some termination criterion $\delta>0$, or $m \geq M_{\text {iter }}$ for some maximum number of iterations $M_{i t e r}$, stop the iterations.

Remark 1. Note that in Algorithm 1 .

- the effective Hamiltonian $H$ satisfies Properties 2 and 6 in Section 1 , i.e.,

$$
H(p, q) \geq 0, \quad \text { and } 1+H(p, q)>u p+v q, \forall(u, v) \in \mathbb{V} .
$$

- the initial guess $H_{0}$ is chosen to satisfy Property 2 in Section 1. With such initial guesses, only a few iterations are needed for convergence to the given termination criterion. To further accelerate the convergence, Newton's method with high order iterations can be easily applied [8].

\subsection{The Method}

With the above numerical techniques and schemes, we summarize the method to solve the kinetic eikonal equation (8), along with equation (9).

Algorithm 2. WENO-RK-Newton methods for equations (8) and (9)

- Compute weights/abscissas of the Gauss quadrature rule, or compute moments of the Maxwellian M.

- For $k=0,1,2, \ldots$ 
- at any node $\left(x_{i}, y_{j}\right)$,

* use the WENO constructions to approximate $\left(\psi_{x}^{ \pm}\right)_{i j}$ and $\left(\psi_{y}^{ \pm}\right)_{i j}$,

* compute the numerical Hamiltonian $\hat{H}\left(\left(\psi_{x}^{+}\right)_{i j},\left(\psi_{x}^{-}\right)_{i j},\left(\psi_{y}^{+}\right)_{i j},\left(\psi_{y}^{-}\right)_{i j}\right)$ by Algorithm 1 .

* use the Runge-Kutta procedures to obtain $\psi_{i j}^{k+1}$,

* Set

$$
\psi_{i j}^{k+1}=\max \left\{0, \psi_{i j}^{k+1}\right\} .
$$

Remark 2. With all the procedures described above, the key properties observed in Section 1 are wellpreserved. The equation (18) in Algorithm 2 is simple, but enough to guarantee that $\psi$ stays nonnegative, without losing accuracy. We may verify it in the following way. Denoting $0=\psi_{\min }=\min \psi_{0}, \psi_{\max }=$ $\max \psi_{0}$, the maximum principle is referred to as: if $\psi_{\min } \leq\left\{\psi_{i j}^{k}\right\} \leq \psi_{\max }$, then $\psi_{\min } \leq\left\{\psi_{i j}^{k+1}\right\} \equiv\left\{\psi_{i j}^{k}-\right.$

$\left.\Delta t \hat{H}\left(\left\{\psi_{i j}^{k}\right\}\right)\right\} \leq \psi_{\max }$, where $\hat{H}$ denotes the numerical Hamiltonian after completing all the stages in the Runge-Kutta procedure.

Since the Hamiltonian $\hat{H} \geq 0$, it is obvious that $\psi_{i j}^{k+1}=\psi_{i j}^{k}-\Delta t \hat{H}\left(\left\{\psi_{i j}^{k}\right\}\right) \leq \psi_{i j}^{k} \leq \psi_{\max }$, i.e., $\left\{\psi_{i j}^{k+1}\right\} \leq$ $\psi_{\max }$. And $\left\{\psi_{i j}^{k+1}\right\} \geq \psi_{\min }=0$ by imposing equation (18). Therefore the maximum principle is satisfied. We further show that enforcing equation (18) maintains the accuracy. Assume the solution is smooth. We show that $\left|\psi_{i j}^{k+1}-\max \left\{\psi_{i j}^{k+1}, 0\right\}\right| \leq \Delta t O\left(h^{P}\right)$, where $P$ is the order of the WENO approximation.

From the kinetic eikonal equation (9), we have

$$
\begin{aligned}
& \int_{\mathbb{V}} \frac{M(\boldsymbol{v})}{1+H(\boldsymbol{p})-\boldsymbol{v} \cdot \boldsymbol{p}} d \boldsymbol{v}=1, \\
\Rightarrow & \int_{\mathbb{V}} \frac{M(\boldsymbol{v})}{1-\frac{\boldsymbol{v} \cdot \boldsymbol{p}}{1+H(\boldsymbol{p})}} d \boldsymbol{v}=1+H(\boldsymbol{p}), \\
\Rightarrow & \int_{\mathbb{V}} M(\boldsymbol{v}) \sum_{m=0}^{\infty}\left(\frac{\boldsymbol{v} \cdot \boldsymbol{p}}{1+H(\boldsymbol{p})}\right)^{m} d \boldsymbol{v}=1+H(\boldsymbol{p}), \\
\Rightarrow & \int_{\mathbb{V}} M(\boldsymbol{v})\left(\frac{\boldsymbol{v} \cdot \boldsymbol{p}}{1+H(\boldsymbol{p})}\right)^{2} d \boldsymbol{v}+\int_{\mathbb{V}} M(\boldsymbol{v}) \sum_{m=3}^{\infty}\left(\frac{\boldsymbol{v} \cdot \boldsymbol{p}}{1+H(\boldsymbol{p})}\right)^{m} d \boldsymbol{v}=H(\boldsymbol{p}),
\end{aligned}
$$

which implies $H(\boldsymbol{p}) \sim \theta^{2} \boldsymbol{p}^{2}$ as $\boldsymbol{p} \rightarrow \mathbf{0}[3]$.

If $\boldsymbol{x}_{i j}=\left(x_{i}, y_{j}\right)$ is a local minimum point, we have $\left|\hat{H}\left(\left\{\psi_{i j}^{k}\right\}\right)\right| \sim \theta^{2}\left|\hat{\boldsymbol{p}}_{i j}^{2}\right| \sim \theta^{2}\left|\hat{\boldsymbol{p}}_{i j}-\mathbf{0}\right|^{2} \sim O\left(h^{2 P}\right)$ with $\hat{\boldsymbol{p}}_{i j}$ the P-th order WENO approximation of $\nabla \psi_{i j}^{k} \sim \mathbf{0}$. If $\psi_{i j}^{k+1} \geq 0,\left|\psi_{i j}^{k+1}-\max \left\{\psi_{i j}^{k+1}, 0\right\}\right|=0$. If $\psi_{i j}^{k+1}<0$, we have $\psi_{i j}^{k} \geq 0>\psi_{i j}^{k+1}$, and $\left|\psi_{i j}^{k+1}-\max \left\{\psi_{i j}^{k+1}, 0\right\}\right|=\left|\psi_{i j}^{k+1}-0\right|<\left|\psi_{i j}^{k}-\psi_{i j}^{k+1}\right|=\left|\Delta t \hat{H}\left(\left\{\psi_{i j}^{k}\right\}\right)\right|$. Therefore, $\left|\psi_{i j}^{k+1}-\max \left\{\psi_{i j}^{k+1}, 0\right\}\right| \leq \Delta t O\left(h^{2 P}\right)$. 
If $\boldsymbol{x}_{i j}=\left(x_{i}, y_{j}\right)$ is close to a local minimum point $\boldsymbol{x}_{*}=\left(x_{*}, y_{*}\right)$, we have, by L'Hospital's Rule,

$$
\begin{aligned}
\lim _{\boldsymbol{x} \rightarrow \boldsymbol{x}_{*}} \frac{x \partial \psi(t, \boldsymbol{x}) / \partial x}{\psi(t, \boldsymbol{x})} & =\lim _{\boldsymbol{x} \rightarrow \boldsymbol{x}_{*}} \frac{\partial \psi(t, \boldsymbol{x}) / \partial x+x \partial^{2} \psi(t, \boldsymbol{x}) / \partial x^{2}}{\partial \psi(t, \boldsymbol{x}) / \partial x} \\
& =\lim _{\boldsymbol{x} \rightarrow \boldsymbol{x}_{*}} \frac{2 \partial^{2} \psi(t, \boldsymbol{x}) / \partial x^{2}+x \partial^{3} \psi(t, \boldsymbol{x}) / \partial x^{3}}{\partial^{2} \psi(t, \boldsymbol{x}) / \partial x^{2}} \\
& =\cdots \\
& =\lim _{\boldsymbol{x} \rightarrow \boldsymbol{x}_{*}} \frac{K \partial^{K} \psi(t, \boldsymbol{x}) / \partial x^{K}+x \partial^{K+1} \psi(t, \boldsymbol{x}) / \partial x^{K+1}}{\partial^{K} \psi(t, \boldsymbol{x}) / \partial x^{K}}=K,
\end{aligned}
$$

where $K$ is the smallest constant such that $\partial^{k} \psi\left(t, \boldsymbol{x}_{*}\right) / \partial x^{k}=0$ for $k \leq K-1$, but $\partial^{K} \psi\left(t, \boldsymbol{x}_{*}\right) / \partial x^{K} \neq 0$. Similarly, we have $\lim _{\boldsymbol{x} \rightarrow \boldsymbol{x}_{*}} \frac{y \partial \psi(t, \boldsymbol{x}) / \partial y}{\psi(t, \boldsymbol{x})}=L$, where $L$ is the smallest constant such that $\partial^{l} \psi\left(t, \boldsymbol{x}_{*}\right) / \partial y^{l}=0$ for $l \leq L-1$, but $\partial^{L} \psi\left(t, \boldsymbol{x}_{*}\right) / \partial y^{L} \neq 0$. Note that the L'Hospital's Rule is repeatedly applied only if it is applicable after necessary cancellations. Therefore, we have $\frac{\|x\|_{\infty}\|\nabla \psi(t, x)\|_{\infty}}{|\psi(t, \boldsymbol{x})|} \leq C$ for some constant $C>0$ as $\boldsymbol{x} \rightarrow \boldsymbol{x}_{*}$. That is, we have $\psi(t, \boldsymbol{x}) \geq \frac{\|\boldsymbol{x}\|_{\infty}\|\nabla \psi(t, \boldsymbol{x})\|_{\infty}}{C}$ as $\boldsymbol{x} \rightarrow \boldsymbol{x}_{*}$. And we know $|H(\nabla \psi(t, \boldsymbol{x}))| \leq V_{\max }\|\nabla \psi(t, \boldsymbol{x})\|_{\infty}$. Hence, if we choose the CFL condition $\Delta t \leq \frac{\max \{\Delta x, \Delta y\}}{2 C V_{\max }}$, then $\psi_{i j}^{k+1}=\psi_{i j}^{k}-\Delta t \hat{H}\left(\left\{\psi_{i j}^{k}\right\}\right) \geq 0$. The constant $C$ may be estimated through the initial condition $\psi_{0}(\boldsymbol{x})$.

Remark 3. Assuming that $O(N)$ mesh points are used in each dimension of $\boldsymbol{x}$, and $T$ abscissas/weights in each dimension of $\boldsymbol{v}$ are used in the Gauss quadrature formula, or $T$ moments of the Maxwellian in each dimension of $\boldsymbol{v}$ are used in the expansions with respect to moments, then at each time step, the total complexity of the method is $T^{n} O\left(N^{n}\right)$. In practice, it is possible to choose $T \ll N$, which assures that the method is efficient. This is the motivation of a forthcoming work which aims to design an effective method for approximating the density $f^{\epsilon}$ of the original BGK equation (1). The complexity of solving the BGK equation (1) is in general of order $O\left(N^{2 n}\right)$ if $O(N)$ points are used in each dimension of $\boldsymbol{x}$ and $\boldsymbol{v}$, which is much higher than $T^{n} O\left(N^{n}\right)$ if $N \gg T$. Following this work, we will design an effective method for approximating $f^{\epsilon}$ with complexity $T^{n} O\left(N^{n}\right)$, which will be discussed in [14].

Remark 4. There are two sources of errors. One is the numerical error in the schemes based on WENORK approximations for general Hamilton-Jacobi equations. The other one is the numerical error in the computation of the effective Hamiltonian by solving the nonlinear equation (10) with Newton's method, which depends on the approximation of the integrals in Algorithm 1. Therefore, the overall error for smooth solutions is expected to be $O\left((\Delta x)^{P}+(\Delta y)^{P}+(\Delta t)^{P}+E^{H a m}\right)$ if a $P$-th order WENO approximation and a P-th order RK procedure are used, where $E^{H a m}$ represents the error in the computation of the effective Hamiltonian by Algorithm 1. Numerical experiments in Section 3 verify the error estimate. 
We present several numerical experiments in the following section to demonstrate the effectiveness of the method.

\section{Numerical Examples}

Several numerical examples are presented to demonstrate the effectiveness of our methods. In all the examples, we choose $\delta=10^{-14}, M_{i t e r}=20$ in Algorithm 1 We use WENO\#-RK\#-Godunov to denote the methods based on WENO5-RK5 or WENO3-RK3, and the Godunov scheme. In all the examples, if a Gauss formula is used, the abscissas/weights are generated by the algorithm in [6] with $M(\mathbf{v})$ as the weight function. The number of abscissas/weights for each dimension used in the Gauss quadrature formula, or the number of moments of the Maxwellian for each dimension used in the expansions with respect to moments, is recorded. Maximum errors between the numerical solutions and the reference solutions at the mesh points are recorded. All the experiments are performed on a MacBook Pro with codes written in $\mathbf{C}$, and double precision is used. Numerical plots are obtained with Matlab.

Example 1: 1-D examples. We fist choose a constant Maxwellian, $M(v)=0.5$ for $\mathbb{V}=[-1,1]$. The computational domain is $\Omega=[-1,1]$, and the initial condition is $\psi(0, x)=0.1(1+\sin (2 \pi(x+1)))$. The effective Hamiltonian is given explicitly as $H(p)=\frac{p-\tanh (p)}{\tanh (p)}$. Table 1 shows the efficiency and accuracy of Algorithm 1 for computing the effective Hamiltonian. Note that moments of the Maxwellian of odd orders are zeros, which can be ignored in computations. Therefore, in Table 1 although the first 10, 20, 40, or 60 moments of the Maxwellian are included, only the first 5,10,20, or 30 moments of even orders are used in computations, respectively. If a Gauss quadrature rule is used, only 16 abscissas/weights are enough to reach the machine precision. Average 4 to 6 iterations are needed for convergence. Figure 1 shows numerical plots of the effective Hamiltonian. The effective Hamiltonian is convex and nonnegative. Table 2 shows the accuracy of Algorithm 2 for computing $\psi$. The reference solution is computed on a refined mesh of size 2561 with exact effective Hamiltonian and the WENO5-RK5-Godunov scheme, without imposing equation (18). The numerical solutions are computed by Algorithm 2 with the Godunov scheme. 8 or 16 abscissas/weights are used in the Gauss quadrature formula, and 15 or 30 moments of the Maxwellian are used in the expansions with respect to moments. The desired order of accuracy is observed until the error of computing the effective Hamiltonian by Algorithm 1 becomes dominant. Figure 2 shows the numerical solutions. It is clear that the positivity of $\psi$ is preserved. Table 3 shows the CPUtime for computing the numerical solution at $t=0.1$. The CPUtime increases quadratically as the mesh size is refined, which verifies that at each time step the complexity is $O(N T)$.

We further choose $M(v)=\frac{e^{-v^{2}}}{\sqrt{\pi} \operatorname{erf}(2)}$ for $\mathbb{V}=[-2,2]$. The computational domain is $\Omega=[-1,1]$, and the initial condition is $\psi(0, x)=0.1(1+\sin (\pi(x+1)))$. Table 4 shows the accuracy of Algorithm 2 


\begin{tabular}{|c|c|c|c|c|}
\hline \# of Gauss Absci. & 4 & 8 & 16 & 32 \\
\hline$\left\|H^{h}-H\right\|_{\infty}$ & $9.165 \mathrm{E}-5$ & $6.938 \mathrm{E}-9$ & $3.192 \mathrm{E}-16$ & $5.412 \mathrm{E}-16$ \\
\hline Ave. \# of Iter. & $4-6$ & $4-6$ & $4-6$ & $4-6$ \\
\hline \hline \# of moments & 10 & 20 & 40 & 60 \\
\hline$\left\|H^{h}-H\right\|_{\infty}$ & $2.767 \mathrm{E}-4$ & $4.261 \mathrm{E}-7$ & $1.827 \mathrm{E}-12$ & $3.089 \mathrm{E}-16$ \\
\hline Ave. \# of Iter. & $4-6$ & $4-6$ & $4-6$ & $4-6$ \\
\hline
\end{tabular}

Table 1: Accuracy of Algorithm 1 for Example 1 with constant Maxwellian: maximum error between the computed effective Hamiltonian $H^{h}\left(\psi_{x}(0, x)\right)$ and the exact effective Hamiltonian $H\left(\psi_{x}(0, x)\right)$ is recorded with different numbers of Gauss abscissas/weights, and different number of moments of the Maxwellian. Average number of iterations used in the Algorithm is recorded.

The reference solution is computed on a refined mesh of size 2561 with 32 Gauss abscissas/weights and WENO5-RK5-Godunov scheme, without imposing equation (18). The desired order of accuracy is observed until the error of computing the effective Hamiltonian by Algorithm 1 becomes dominant. Figure 3 shows the numerical solutions. It is clear that the positivity of $\psi$ is preserved. Table 5 shows the CPUtime for computing the numerical solution at $t=0.1$. The CPUtime increases quadratically as the mesh size is refined, which verifies that at each time step the complexity is $O(N T)$.

Example 2: 2-D example. We choose $M(u, v)=\frac{e^{-\left(u^{2}+v^{2}\right)}}{\pi \operatorname{erf}^{2}(2)}$ for $\mathbb{V}=[-2,2]^{2}$. The computational domain is $\Omega=[-1,1]^{2}$. The initial condition is $\psi(0, x, y)=0.025(2-\sin (2 \pi(x+1))-\cos (2 \pi(y+1)))$. Table 6 shows the accuracy of the effective Hamiltonian computed by Algorithm 1. Figure 4 shows the effective Hamiltonian computed by Algorithm 1. It is clear that the effective Hamiltonian is convex and nonnegative. Table 7 shows the accuracy of Algorithm 2 for computing $\psi$. The reference solution is computed by WENO5-RK5-Godunov on a mesh of size $321 \times 321$ with 32 abscissas/weights, without imposing equation (18). The numerical solutions are computed by WENO-RK-Godunov with 16 or 8 abscissas/weights or 30 or 15 moments of the Maxwellian. The desired order of accuracy is observed until the error of computing the effective Hamiltonian by Algorithm 1 becomes dominant. Figure 5 shows the numerical solutions. It is clear that $\psi$ is nonnegative. In the expansions with respect to moments of the Maxwellian, only moments of even orders are used in computations. Table 8 shows the CPUtime for computing the numerical solution at $t=0.1$. The CPUtime increases cubically as the mesh size is refined, which verifies that at each time step the complexity is $O\left(N^{2} T^{2}\right)$.

Example 3: 3-D example. We choose $M(\mathbf{v})=\frac{e^{-\mathbf{v}^{2}}}{\pi^{3 / 2} \operatorname{erf}^{3}(2)}$ for $\mathbb{V}=[-2,2]^{3}$. The computational domain is $\Omega=[-1,1]^{3}$. The initial condition is $\psi(0, \mathbf{x})=0.025(3-\sin (2 \pi(x+1))-\cos (2 \pi(y+1))-\sin (2 \pi(z+1)))$. Table 9 shows the accuracy of the effective Hamiltonian computed by Algorithm 1. Figure 6 shows the effective Hamiltonian computed by Algorithm 1. It is clear that the effective Hamiltonian is convex and nonnegative. Figure 7 shows the numerical solutions computed by Algorithm 2 with Godunov scheme on a mesh of size $41 \times 41 \times 41$. 8 Gauss abscissas/weights are used. It is clear that $\psi$ is nonnegative. In 

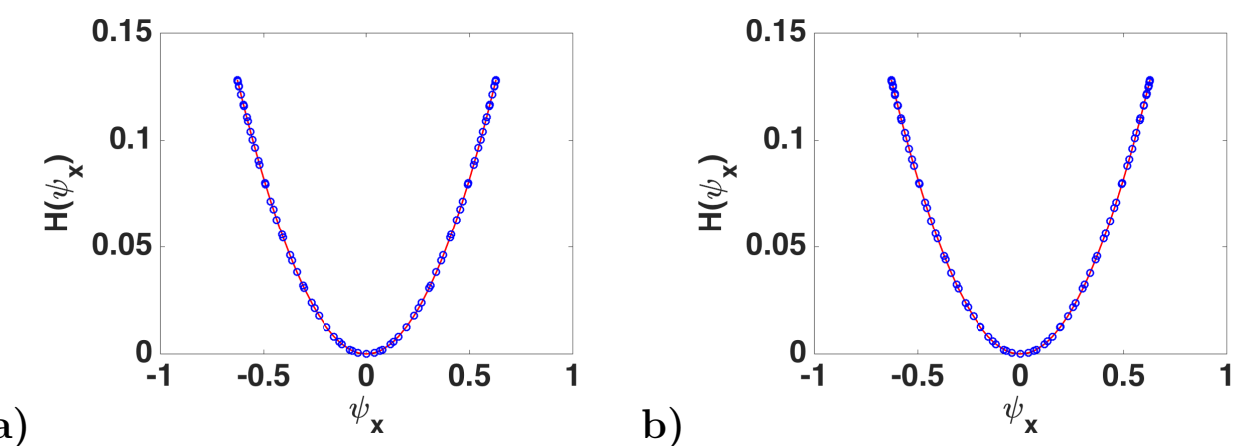

a)
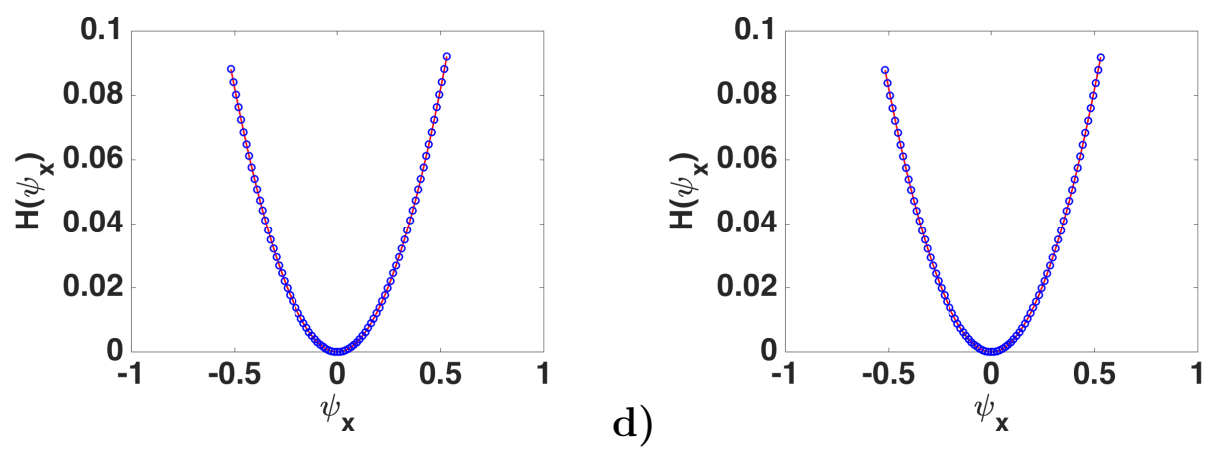

Figure 1: Effective Hamiltonian for Example 1 with constant Maxwellian: mesh for $x$ is 161 . Solid line: exact effective Hamiltonian; Circles: computed effective Hamiltonian by Algorithm 1 (a) and (b): at $t=0.1$; (c) and (d): at $t=1$. Left: with WENO3 ; Right: with WENO5.

the expansions with respect to moments of the Maxwellian, only moments of even orders are used in the computations.

Remark 5. We remark that since the Maxwellian $M(v)$ is independent of $t$, one can pre-compute a table of the effective Hamiltonian $H$ on a refined mesh. Then at each time step, one can read this table with appropriate interpolations to obtain the effective Hamiltonian $H$ at any point without applying Algorithm 1 to compute $H$ again. Therefore, the computational complexity at each time step is reduced to $O\left(N^{n}\right)$, which implies that less CPUtime is needed for the computation. Table 10 shows the CPUtime for computing the numerical solution at $t=0.1$ with pre-computed effective Hamiltonian with Example 1. The CPUtime increases quadratically as the mesh size is refined, which verifies that at each time step the complexity is $O(N)$, and the amount of CPUtime is much less than that listed in Table 5 Table 11 shows the CPUtime for computing the numerical solution at $t=0.1$ with pre-computed effective Hamiltonian with Example 2. The CPUtime increases cubically as the mesh size is refined, which verifies that at each time step the complexity is $O\left(N^{2}\right)$, and the amount of CPUtime is much less than that listed in Table 8 . For Maxwellian $M$ that depends on $t$, this approach is not applicable. 


\begin{tabular}{|c|c|c|c|c|c|}
\hline \multicolumn{6}{|c|}{ WENO5-RK5-Godunov, 16 Gauss abscissas/weights } \\
\hline Mesh & 21 & 41 & 81 & 161 & 321 \\
\hline$\left\|\psi^{h}-\psi^{\text {ref }}\right\|_{\infty}$ & $2.609 \mathrm{E}-5$ & $2.446 \mathrm{E}-6$ & $8.997 \mathrm{E}-8$ & $3.012 \mathrm{E}-9$ & $9.669 \mathrm{E}-11$ \\
\hline Order of Conv. & - & 3.42 & 4.76 & 4.90 & 4.96 \\
\hline \hline \multicolumn{6}{|c|}{ WENO3-RK3-Godunov, 16 Gauss abscissas/weights } \\
\hline Mesh & 21 & 41 & 81 & 161 & 321 \\
\hline$\left\|\psi^{h}-\psi^{\text {ref }}\right\|_{\infty}$ & $1.770 \mathrm{E}-3$ & $6.220 \mathrm{E}-4$ & $5.639 \mathrm{E}-5$ & $1.305 \mathrm{E}-6$ & $7.839 \mathrm{E}-8$ \\
\hline Order of Conv. & - & 1.51 & 3.46 & 5.43 & 4.06 \\
\hline \hline \multicolumn{6}{|c|}{ WENO5-RK5-Godunov, 8 Gauss abscissas/weights } \\
\hline Mesh & 21 & 41 & 81 & 161 & 321 \\
\hline$\left\|\psi^{h}-\psi^{\text {ref }}\right\|_{\infty}$ & $2.609 \mathrm{E}-5$ & $2.446 \mathrm{E}-6$ & $8.997 \mathrm{E}-8$ & $3.017 \mathrm{E}-9$ & $7.268 \mathrm{E}-10$ \\
\hline Order of Conv. & - & 3.42 & 4.76 & 4.90 & $\mathbf{2 . 0 5}$ \\
\hline \hline \multicolumn{6}{|c|}{ WENO3-RK3-Godunov, 8 Gauss abscissas/weights } \\
\hline Mesh & 21 & 41 & 81 & 161 & 321 \\
\hline$\left\|\psi^{h}-\psi^{\text {ref }}\right\|_{\infty}$ & $1.770 \mathrm{E}-3$ & $6.229 \mathrm{E}-4$ & $5.633 \mathrm{E}-5$ & $1.301 \mathrm{E}-6$ & $7.853 \mathrm{E}-8$ \\
\hline Order of Conv. & - & 1.51 & 3.47 & 5.44 & 4.05 \\
\hline \hline \hline
\end{tabular}

\begin{tabular}{|c|c|c|c|c|c|}
\hline \hline \multicolumn{6}{|c|}{ WENO5-RK5-Godunov, 30 moments of the Maxwellian } \\
\hline Mesh & 21 & 41 & 81 & 161 & 321 \\
\hline$\left\|\psi^{h}-\psi^{\text {ref }}\right\|_{\infty}$ & $2.609 \mathrm{E}-5$ & $2.446 \mathrm{E}-6$ & $8.997 \mathrm{E}-8$ & $3.012 \mathrm{E}-9$ & $1.176 \mathrm{E}-10$ \\
\hline Order of Conv. & - & 3.42 & 4.76 & 4.90 & $\mathbf{4 . 6 8}$ \\
\hline \hline \multicolumn{6}{|c|}{ WENO3-RK3-Godunov, 30 moments of the Maxwellian } \\
\hline Mesh & 21 & 41 & 81 & 161 & 321 \\
\hline$\left\|\psi^{h}-\psi^{\text {ref }}\right\|_{\infty}$ & $1.770 \mathrm{E}-3$ & $6.220 \mathrm{E}-4$ & $5.639 \mathrm{E}-5$ & $1.305 \mathrm{E}-6$ & $7.839 \mathrm{E}-8$ \\
\hline Order of Conv. & - & 1.51 & 3.46 & 5.43 & 4.06 \\
\hline \hline \multicolumn{6}{|c|}{ WENO5-RK5-Godunov, 15 moments of the Maxwellian } \\
\hline Mesh & 21 & 41 & 81 & 161 & 321 \\
\hline$\left\|\psi^{h}-\psi^{\text {ref }}\right\|_{\infty}$ & $2.651 \mathrm{E}-5$ & $2.458 \mathrm{E}-6$ & $5.516 \mathrm{E}-7$ & $5.435 \mathrm{E}-7$ & $5.425 \mathrm{E}-7$ \\
\hline Order of Conv. & - & $\mathbf{3 . 4 3}$ & $\mathbf{2 . 1 6}$ & $\mathbf{0 . 0 2}$ & $\mathbf{0 . 0 0 3}$ \\
\hline \hline \multicolumn{6}{|c|}{ WENO3-RK3-Godunov, 15 moments of the Maxwellian } \\
\hline Mesh & 21 & 41 & 81 & 161 & 321 \\
\hline$\left\|\psi^{h}-\psi^{\text {ref }}\right\|_{\infty}$ & $1.770 \mathrm{E}-3$ & $6.227 \mathrm{E}-4$ & $5.626 \mathrm{E}-5$ & $1.294 \mathrm{E}-6$ & $5.883 \mathrm{E}-7$ \\
\hline Order of Conv. & - & 1.51 & 3.47 & 5.44 & $\mathbf{1 . 1 4}$ \\
\hline
\end{tabular}

Table 2: Accuracy of Algorithm 2 for Example 1 with constant Maxwellian: maximum error between the numerical solution $\psi^{h}(0.1, x)$ by Algorithm 2 with Godunov scheme and the reference solution $\psi^{r e f}(0.1, x) .16$ or 8 Gauss abscissas/weights are used in the Gauss quadrature formula, and 30 or 15 moments of the Maxwellian are used in the expansions with respect to moments. The desired order of accuracy is observed until the error of computing the effective Hamiltonian by Algorithm 1 becomes dominant (marked as bold numbers).

\begin{tabular}{|c|c|c|c|c|c|}
\hline Mesh & 101 & 201 & 401 & 801 & 1601 \\
\hline \multicolumn{7}{|c|}{16 Gauss abscissas/weights } \\
\hline CPUtime (sec.) & $1.441 \mathrm{E}-2$ & $3.567 \mathrm{E}-2$ & $1.265 \mathrm{E}-1$ & $4.348 \mathrm{E}-1$ & $1.650 \mathrm{E} 0$ \\
\hline \multicolumn{7}{|c|}{30 moments of the Maxwellian } \\
\hline CPUtime (sec.) & $2.067 \mathrm{E}-2$ & $6.029 \mathrm{E}-2$ & $2.147 \mathrm{E}-1$ & $8.011 \mathrm{E}-1$ & $3.245 \mathrm{E} 0$ \\
\hline
\end{tabular}

Table 3: CPUtime for computing the numerical solution for Example 1 with constant Maxwellian at $t=0.1$. 
a)
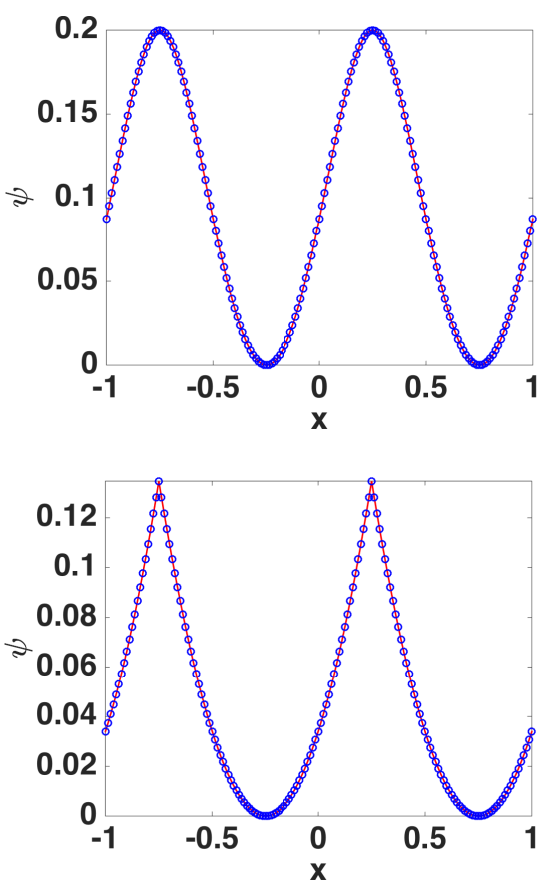

b)

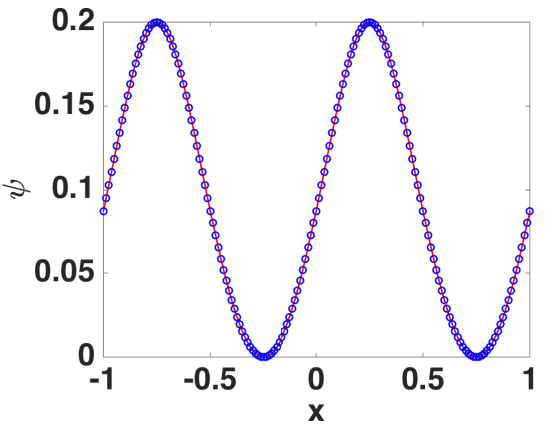

d)

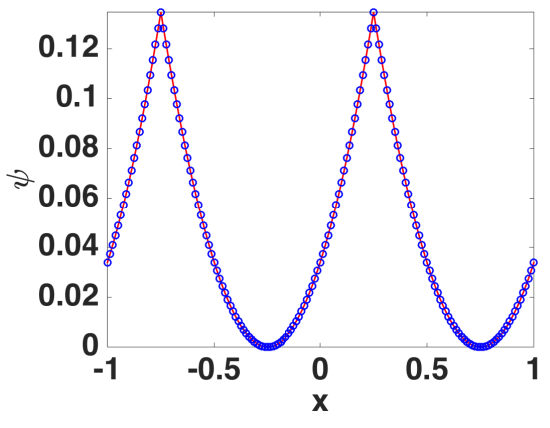

Figure 2: Numerical solutions for Example 1 with constant Maxwellian: mesh 161. Solid line: reference solution; Circles: computed solution by Algorithm 2 (a) and (b): at $t=0.1$; (c) and (d): at $t=1$. Left: with WENO3-RK3-Godunov ; Right: with WENO5-RK5-Godunov.

\section{Conclusions}

We present efficient high order finite difference numerical methods based on the WENO constructions and the Runge-Kutta procedures for solving the kinetic eikonal equation, where the effective Hamiltonian is computed through the solution of a nonlinear equation that is solved by Newton's method. During Newton's iterations, integrals with respect to the velocity variables are evaluated either by a Gauss quadrature formula, or as expansions with respect to moments of the Maxwellian. The methods are designed such that the positivity of the viscosity solution and the positivity of the effective Hamiltonian are well-preserved. The complexity of the proposed methods is $T^{n} O\left(N^{n}\right)$ at each time step where $T$ is the number of Gauss abscissas/weights or moments of the Maxwellian, and $N$ is the number of points used in each space dimension. $T$ can be chosen to be much smaller than $N$. Numerical examples verify the effectiveness of the proposed methods.

Efficient computation of $\psi$ with key properties preserved enables us to further design effective numerical methods for approximating the phase function of the Hopf-Cole transformation, hence the density $f^{\epsilon}$. In practice, $\psi$ is not enough to approximate the density $f^{\epsilon}$ with the Hopf-Cole transformation. Motivated by the WKB geometrical-optics approximations in high frequency wave propagation, higher order terms beyond $\psi$ must be included to approximate the phase function, hence the density $f^{\epsilon}$ faithfully. Higher order terms 


\begin{tabular}{|c|c|c|c|c|c|}
\hline \multicolumn{6}{|c|}{ WENO5-RK5-Godunov, 16 Gauss abscissas/weights } \\
\hline Mesh & 21 & 41 & 81 & 161 & 321 \\
\hline$\left\|\psi^{h}-\psi^{r e f}\right\|_{\infty}$ & $5.625 \mathrm{E}-7$ & $2.177 \mathrm{E}-8$ & $7.451 \mathrm{E}-10$ & $2.383 \mathrm{E}-11$ & $7.494 \mathrm{E}-13$ \\
\hline Order of Conv. & - & 4.69 & 4.87 & 4.97 & 4.99 \\
\hline \multicolumn{6}{|c|}{ WENO3-RK3-Godunov, 16 Gauss abscissas/weights } \\
\hline Mesh & 21 & 41 & 81 & 161 & 321 \\
\hline$\left\|\psi^{h}-\psi^{r e f}\right\|_{\infty}$ & $2.662 \mathrm{E}-4$ & $2.085 \mathrm{E}-5$ & $4.588 \mathrm{E}-7$ & $2.841 \mathrm{E}-8$ & $3.371 \mathrm{E}-9$ \\
\hline Order of Conv. & - & 3.67 & 5.50 & 4.01 & 3.08 \\
\hline \multicolumn{6}{|c|}{$\begin{array}{l}\text { WENO5-RK5-Godunov, } 8 \text { Gauss abscissas/weights } \\
\end{array}$} \\
\hline Mesh & 21 & 41 & 81 & 161 & 321 \\
\hline$\left\|\psi^{h}-\psi^{r e f}\right\|_{\infty}$ & $5.630 \mathrm{E}-7$ & $2.257 \mathrm{E}-8$ & $1.547 \mathrm{E}-9$ & $8.452 \mathrm{E}-10$ & $8.239 \mathrm{E}-10$ \\
\hline Order of Conv. & - & 4.65 & 3.87 & 0.87 & 0.04 \\
\hline \multicolumn{6}{|c|}{ WENO3-RK3-Godunov, 8 Gauss abscissas/weights } \\
\hline Mesh & 21 & 41 & 81 & 161 & 321 \\
\hline$\left\|\psi^{h}-\psi^{r e f}\right\|_{\infty}$ & $2.669 \mathrm{E}-4$ & $2.084 \mathrm{E}-5$ & $4.579 \mathrm{E}-7$ & $2.839 \mathrm{E}-8$ & $3.368 \mathrm{E}-9$ \\
\hline Order of Conv. & - & 3.67 & 5.51 & 4.01 & 3.08 \\
\hline \multicolumn{6}{|c|}{ WENO5-RK5-Godunov, 30 moments of the Maxwellian } \\
\hline Mesh & 21 & 41 & 81 & 161 & 321 \\
\hline$\left\|\psi^{h}-\psi^{r e f}\right\|_{\infty}$ & $5.625 \mathrm{E}-7$ & $2.182 \mathrm{E}-8$ & $7.926 \mathrm{E}-10$ & $7.279 \mathrm{E}-11$ & $5.022 \mathrm{E}-11$ \\
\hline Order of Conv. & - & 4.69 & 4.78 & 3.44 & 0.54 \\
\hline \multicolumn{6}{|c|}{ WENO3-RK3-Godunov, 30 moments of the Maxwellian } \\
\hline Mesh & 21 & 41 & 81 & 161 & 321 \\
\hline$\left\|\psi^{h}-\psi^{r e f}\right\|_{\infty}$ & $2.662 \mathrm{E}-4$ & $2.085 \mathrm{E}-5$ & $4.589 \mathrm{E}-7$ & $2.841 \mathrm{E}-8$ & $3.371 \mathrm{E}-9$ \\
\hline Order of Conv. & - & 3.67 & 5.51 & 4.01 & 3.08 \\
\hline \multicolumn{6}{|c|}{ WENO5-RK5-Godunov, 15 moments of the Maxwellian } \\
\hline Mesh & 21 & 41 & 81 & 161 & 321 \\
\hline$\left\|\psi^{h}-\psi^{r e f}\right\|_{\infty}$ & $6.616 \mathrm{E}-7$ & $1.626 \mathrm{E}-7$ & $1.444 \mathrm{E}-7$ & $1.442 \mathrm{E}-7$ & $1.443 \mathrm{E}-7$ \\
\hline Order of Conv. & - & 2.025 & 0.171 & 0.002 & $\begin{array}{l}-0.001 \\
\end{array}$ \\
\hline \multicolumn{6}{|c|}{ WENO3-RK3-Godunov, 15 moments of the Maxwellian } \\
\hline Mesh & 21 & 41 & 81 & 161 & 321 \\
\hline$\left\|\psi^{h}-\psi^{r e f}\right\|_{\infty}$ & $2.669 \mathrm{E}-4$ & $2.079 \mathrm{E}-5$ & $4.519 \mathrm{E}-7$ & $1.554 \mathrm{E}-7$ & $1.456 \mathrm{E}-7$ \\
\hline Order of Conv. & - & 3.68 & 5.52 & 1.54 & 0.09 \\
\hline
\end{tabular}

Table 4: Accuracy of Algorithm2 for Example 1 with non-constant Maxwellian: maximum error between the numerical solution $\psi^{h}(0.1, x)$ by Algorithm 2 with Godunov scheme and the reference solution $\psi^{r e f}(0.1, x)$. 16 or 8 Gauss abscissas/weights are used in the Gauss quadrature formula, and 30 or 15 moments of the Maxwellian are used in the expansions with respect to moments. The desired order of accuracy is observed until the error of computing the effective Hamiltonian by Algorithm 1 becomes dominant (marked as bold numbers).

\begin{tabular}{|c|c|c|c|c|c|}
\hline Mesh & 101 & 201 & 401 & 801 & 1601 \\
\hline \multicolumn{7}{|c|}{16 Gauss abscissas/weights } \\
\hline CPUtime (sec.) & $1.184 \mathrm{E}-2$ & $3.473 \mathrm{E}-2$ & $1.116 \mathrm{E}-1$ & $4.079 \mathrm{E}-1$ & $1.544 \mathrm{E} 0$ \\
\hline \multicolumn{7}{|c|}{30 moments of the Maxwellian } \\
\hline CPUtime (sec.) & $1.732 \mathrm{E}-2$ & $5.009 \mathrm{E}-2$ & $1.750 \mathrm{E}-1$ & $6.456 \mathrm{E}-1$ & $2.521 \mathrm{E} 0$ \\
\hline
\end{tabular}

Table 5: CPUtime for computing the numerical solution for Example 1 with non-constant Maxwellian at $t=0.1$. 
a)

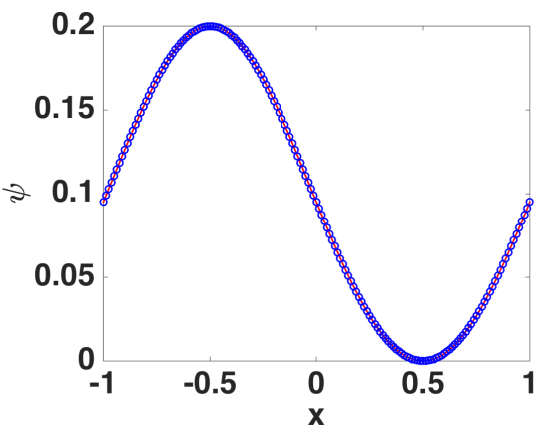

c)

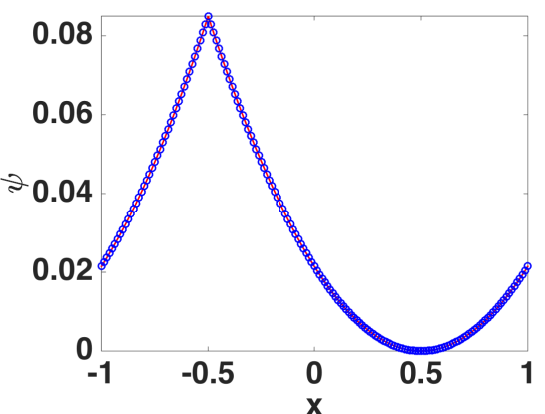

b)

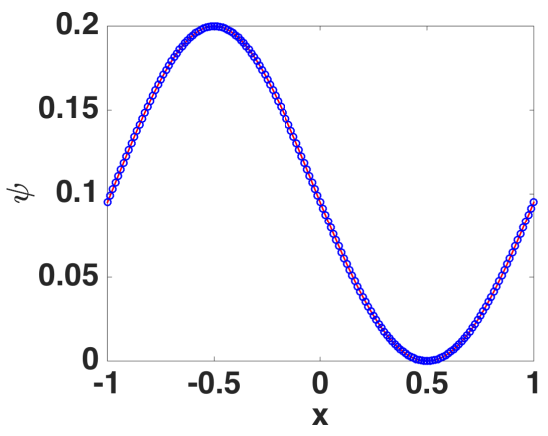

d)

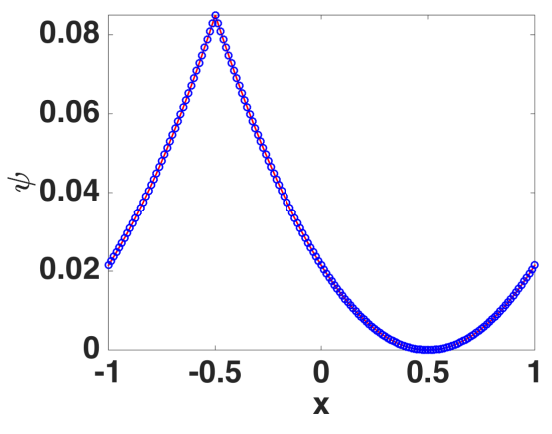

Figure 3: Numerical solutions for Example 1 with non-constant Maxwellian: mesh 161. Solid line: reference solution; Circles: computed solution by Algorithm 2 (a) and (b): at $t=0.1$; (c) and (d): at $t=5$. Left: with WENO3-RK3-Godunov ; Right: with WENO5-RK5-Godunov.

\begin{tabular}{|c|c|c|c|c|}
\hline \# of Gauss Absci. & 4 & 8 & 16 & 32 \\
\hline$\left\|H^{h}-H\right\|_{\infty}$ & $4.422 \mathrm{E}-7$ & $6.423 \mathrm{E}-13$ & $3.109 \mathrm{E}-15$ & $3.109 \mathrm{E}-15$ \\
\hline Ave. \# of Iter. & $4-6$ & $4-6$ & $4-6$ & $4-6$ \\
\hline \hline \# of moments & 10 & 20 & 40 & 60 \\
\hline$\left\|H^{h}-H\right\|_{\infty}$ & $3.252 \mathrm{E}-6$ & $1.798 \mathrm{E}-9$ & $9.325 \mathrm{E}-15$ & $1.998 \mathrm{E}-15$ \\
\hline Ave. \# of Iter. & 4 & 4 & 4 & 2 \\
\hline
\end{tabular}

Table 6: Accuracy of Algorithm 1 for Example 2: maximum error between the computed effective Hamiltonian $H^{h}\left(\psi_{x}(0, x, y), \psi_{y}(0, x, y)\right)$ and the reference effective Hamiltonian $H\left(\psi_{x}(0, x, y), \psi_{y}(0, x, y)\right)$ is recorded with different numbers of Gauss abscissas/weights, and different number of moments of the Maxwellian. Average number of iterations used in the Algorithm is recorded. The reference effective Hamiltonian is computed with 64 Gauss-Legendre abscissas/weights.

will depend on the leading order term $\psi$. The overall complexity is expected to be $T^{n} O\left(N^{n}\right)$, which is much lower than the complexity $O\left(N^{2 n}\right)$ for general methods for the BGK equation. Detailed work will be discussed in [14.

\section{References}

[1] M. Bardi and S. J. Osher, The nonconvex multi-dimensional Riemann problem for Hamilton-Jacobi equations, SIAM J. Math. Analy. 22 (1991), 344-351. 


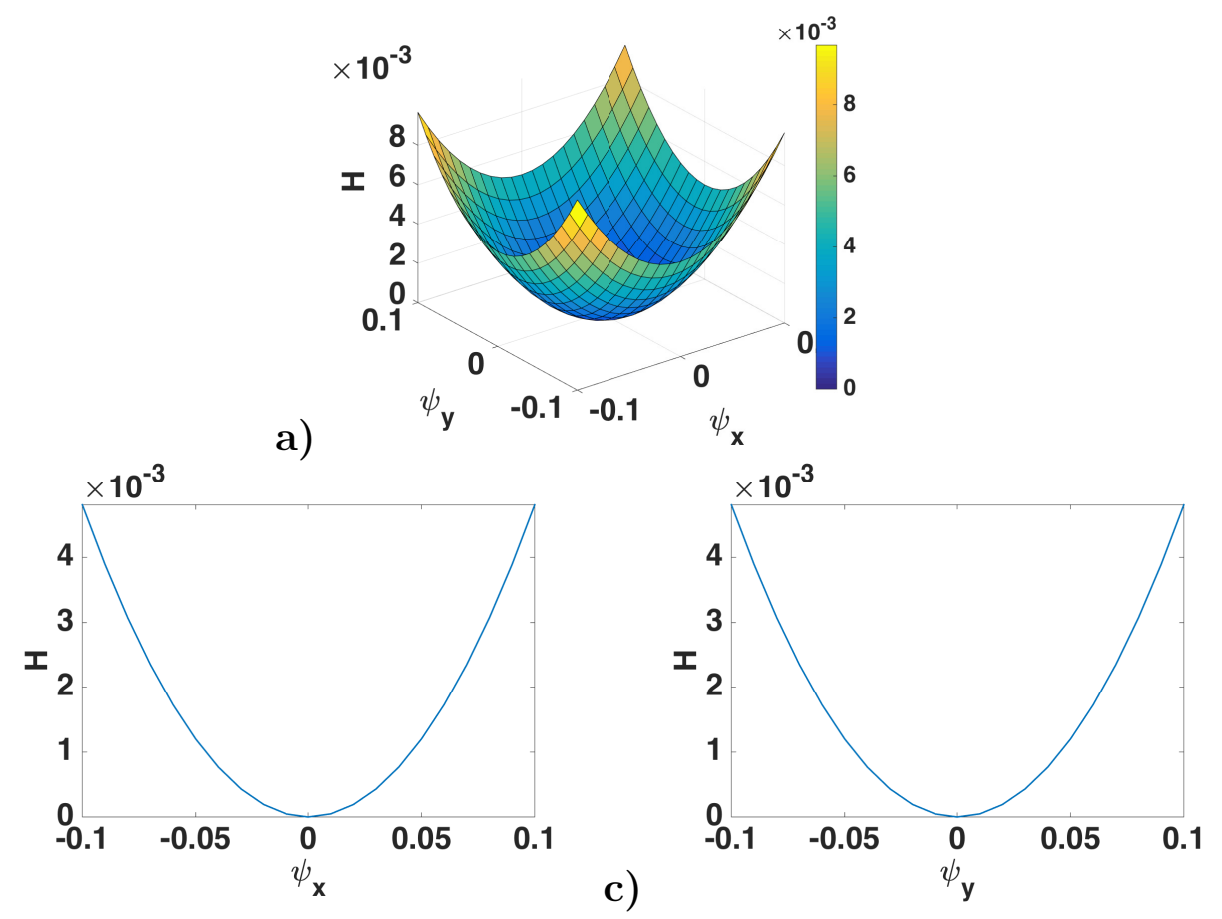

Figure 4: Effective Hamiltonian for Example 2: mesh $21 \times 21$ for $\left(\psi_{x}, \psi_{y}\right)$. The effective Hamiltonian is computed by Algorithm 1 where 16 abscissas/weights are used in the Gauss quadrature formula. (a) surface plot of the effective Hamiltonian; (b) slice at $\psi_{x}=0$; and (c) slice at $\psi_{y}=0$.

[2] P. L. Bhatnagar, E. P. Gross, and M. Krook, A Model for Collision Processes in Gases. I. Small Amplitude Processes in Charged and Neutral One-Component Systems, Phys. Rev. 94 (1954), 511-525.

[3] E. Bouin and V. Calvez, A kinetic eikonal equation, C. R. Acad. Sci. Paris, Ser. I 350 (2012), 243-248.

[4] J. C. Butcher, The numerical analysis of ordinary differential equations, Wiley, New York, 1992.

[5] M. G. Crandall and P.-L. Lions, Two approximations of solutions of Hamilton-Jacobi equations, Math. Comput. 43 (1984), 1-19.

[6] G. H. Golub and J. H. Welsch, Calculation of gauss quadrature rules, Mathematics of Computation 23 (1969), no. 106, 221-s10.

[7] S. Gottlieb, C.-W. Shu, and E. Tadmor, Strong stability preserving high order time discretization methods, SIAM Rev. 43 (2001), 89-112.

[8] A. S. Householder, The Numerical Treatment of a Single Nonlinear Equation, McGraw-Hill, 1970.

[9] G.-S. Jiang and D. Peng, Weighted ENO schemes for Hamilton-Jacobi equations, SIAM J. Sci. Comput. 21 (2000), 2126-2143. 


\begin{tabular}{|c|c|c|c|c|}
\hline \multicolumn{5}{|c|}{ WENO5-RK5-Godunov, 16 Gauss abscissas/weights } \\
\hline Mesh & 21 & 41 & 81 & 161 \\
\hline$\left\|\psi^{h}-\psi^{r e f}\right\|_{\infty}$ & $5.175 \mathrm{E}-6$ & $1.714 \mathrm{E}-7$ & $6.385 \mathrm{E}-9$ & $2.037 \mathrm{E}-10$ \\
\hline Order of Conv. & - & 4.92 & 4.75 & 4.97 \\
\hline \multicolumn{5}{|c|}{ "WENO3-RK3-Godunov, 16 Gauss abscissas/weights } \\
\hline Mesh & 21 & 41 & 81 & 161 \\
\hline$\psi^{h}-\psi^{r e f} \|_{\infty}$ & $4.079 \mathrm{E}-4$ & $6.924 \mathrm{E}-5$ & $1.691 \mathrm{E}-6$ & $1.057 \mathrm{E}-7$ \\
\hline Order of Conv. & - & 2.56 & 5.35 & 4.00 \\
\hline \multicolumn{5}{|c|}{ " WENO5-RK5-Godunov, 8 Gauss abscissas/weights } \\
\hline Mesh & 21 & 41 & 81 & 161 \\
\hline$\left\|\psi^{h}-\psi^{r e f}\right\|_{\infty}$ & $5.175 \mathrm{E}-6$ & $1.715 \mathrm{E}-7$ & $6.385 \mathrm{E}-9$ & $2.037 \mathrm{E}-10$ \\
\hline Order of Conv. & - & 4.92 & 4.75 & 4.97 \\
\hline \multicolumn{5}{|c|}{ WENO3-RK3-Godunov, 8 Gauss abscissas/weights } \\
\hline Mesh & 21 & 41 & 81 & 161 \\
\hline$\left\|\psi^{h}-\psi^{r e f}\right\|_{\infty}$ & $4.079 \mathrm{E}-4$ & $6.924 \mathrm{E}-5$ & $1.691 \mathrm{E}-6$ & $1.057 \mathrm{E}-7$ \\
\hline Order of Conv. & - & 2.56 & 5.35 & 4.00 \\
\hline \multicolumn{5}{|c|}{ WENO5-RK5-Godunov, 30 moments of the Maxwellian } \\
\hline Mesh & 21 & 41 & 81 & 161 \\
\hline $\mid \psi^{h}-\psi^{r e f} \|_{\infty}$ & $5.175 \mathrm{E}-6$ & $1.714 \mathrm{E}-7$ & $6.385 \mathrm{E}-9$ & $2.043 \mathrm{E}-10$ \\
\hline Order of Conv. & - & 4.92 & 4.75 & 4.97 \\
\hline \multicolumn{5}{|c|}{ WENO3-RK3-Godunov, 30 moments of the Maxwellian } \\
\hline Mesh & 21 & 41 & 81 & 161 \\
\hline$\left\|\psi^{h}-\psi^{r e f}\right\|_{\infty}$ & $4.079 \mathrm{E}-4$ & $6.924 \mathrm{E}-5$ & $1.691 \mathrm{E}-6$ & $1.057 \mathrm{E}-7$ \\
\hline Order of Conv. & - & 2.56 & 5.35 & 4.00 \\
\hline \multicolumn{5}{|c|}{ WENO5-RK5-Godunov, 15 moments of the Maxwellian } \\
\hline Mesh & 21 & 41 & 81 & 161 \\
\hline$\left\|\psi^{h}-\psi^{r e f}\right\|_{\infty}$ & $5.175 \mathrm{E}-6$ & $1.744 \mathrm{E}-7$ & $1.053 \mathrm{E}-8$ & $4.511 \mathrm{E}-9$ \\
\hline Order of Conv. & - & 4.89 & 4.04 & 1.22 \\
\hline \multicolumn{5}{|c|}{ WENO3-RK3-Godunov, 15 moments of the Maxwellian } \\
\hline Mesh & 21 & 41 & 81 & 161 \\
\hline$\left\|\psi^{h}-\psi^{r e f}\right\|_{\infty}$ & $4.079 \mathrm{E}-4$ & $6.924 \mathrm{E}-5$ & $1.691 \mathrm{E}-6$ & $1.057 \mathrm{E}-7$ \\
\hline Order of Conv. & - & 2.56 & 5.35 & 4.00 \\
\hline
\end{tabular}

Table 7: Accuracy of Algorithm 2 for Example 2: maximum error between the computed solution $\psi^{h}(0.1, x, y)$ by Algorithm 2 with Godunov scheme and the reference solution $\psi^{r e f}(0.1, x, y) .16$ or 8 Gauss abscissas/weights are used in the Gauss quadrature formula, and 30 or 15 moments of the Maxwellian are used in the expansions with respect to moments. The desired order of accuracy is observed until the error of computing the effective Hamiltonian by Algorithm 1 becomes dominant (marked as bold numbers).

\begin{tabular}{|c|c|c|c|c|}
\hline Mesh & $21 \times 21$ & $41 \times 41$ & $811 \times 81$ & $161 \times 161$ \\
\hline \multicolumn{5}{|c|}{8 Gauss abscissas/weights } \\
\hline CPUtime (sec.) & $2.421 \mathrm{E}-2$ & $1.471 \mathrm{E}-1$ & $1.147 \mathrm{E} 0$ & $9.004 \mathrm{E} 0$ \\
\hline \multicolumn{5}{|c|}{15 moments of the Maxwellian } \\
\hline CPUtime (sec.) & $1.490 \mathrm{E}-2$ & $9.262 \mathrm{E}-2$ & $6.908 \mathrm{E}-1$ & $5.617 \mathrm{E} 0$ \\
\hline
\end{tabular}

Table 8: CPUtime for computing the numerical solution for Example 2 at $t=0.1$. 


\begin{tabular}{|c|c|c|c|c|}
\hline \# of Gauss Absci. & 4 & 8 & 16 & 32 \\
\hline$\left\|H^{h}-H\right\|_{\infty}$ & $1.419 \mathrm{E}-6$ & $4.748 \mathrm{E}-11$ & $3.131 \mathrm{E}-14$ & $3.908 \mathrm{E}-14$ \\
\hline Ave. \# of Iter. & $4-5$ & $4-5$ & $4-5$ & $4-5$ \\
\hline \hline \# of moments & 10 & 20 & 40 & 60 \\
\hline$\left\|H^{h}-H\right\|_{\infty}$ & $3.268 \mathrm{E}-5$ & $3.248 \mathrm{E}-7$ & $1.410 \mathrm{E}-9$ & $2.996 \mathrm{E}-11$ \\
\hline Ave. \# of Iter. & $4-5$ & $4-5$ & $4-5$ & $4-5$ \\
\hline
\end{tabular}

Table 9: Accuracy of Algorithm 1 for Example 3: the maximum error between the computed effective Hamiltonian $H^{h}\left(\nabla_{\mathbf{x}} \psi(0, \mathbf{x})\right)$ and the reference effective Hamiltonian $H\left(\nabla_{\mathbf{x}} \psi(0, \mathbf{x})\right)$ is recorded with different numbers of Gauss abscissas/weights, and different number of moments of the Maxwellian. Average number of iterations used in the Algorithm is recorded. The reference effective Hamiltonian is computed with 64 Gauss abscissas/weights.

\begin{tabular}{|c|c|c|c|c|c|}
\hline Mesh & 101 & 201 & 401 & 801 & 1601 \\
\hline CPUtime (sec.) & $1.035 \mathrm{E}-2$ & $1.967 \mathrm{E}-2$ & $4.316 \mathrm{E}-2$ & $1.346 \mathrm{E}-1$ & $4.631 \mathrm{E}-1$ \\
\hline
\end{tabular}

Table 10: CPUtime for computing the numerical solution for Example 1 with non-constant Maxwellian at $t=0.1$. Table of effective Hamiltonian is pre-computed on a refined mesh with mesh size $5 \times 10^{-4}$ (with CPUtime $2.266 \mathrm{E}-3$ seconds), and 5 th order polynomial interpolation is applied for reading the table. 16 Gauss abscissas/weights are used in the quadrature rule.

\begin{tabular}{|c|c|c|c|c|}
\hline Mesh & $21 \times 21$ & $41 \times 41$ & $811 \times 81$ & $161 \times 161$ \\
\hline CPUtime (sec.) & $4.900 \mathrm{E}-3$ & $2.670 \mathrm{E}-2$ & $1.856 \mathrm{E}-1$ & $1.481 \mathrm{E} 0$ \\
\hline
\end{tabular}

Table 11: CPUtime for computing the numerical solution for Example 2 at $t=0.1$. Table of effective Hamiltonian is precomputed on a refined mesh with mesh size $5 \times 10^{-4}$ (with CPUtime 3.184 seconds), and 5th order polynomial interpolation is applied for reading the table. 8 Gauss abscissas/weights are used in the quadrature rule.

[15] S. Osher and C.-W. Shu, High-order essentially nonoscillatory schemes for Hamilton-Jacobi equations, SIAM J. Numer. Anal. 28 (1991), no. 4, 907-922.

[16] L. Pareschi and G. Russo, Numerical Solution of the Boltzmann Equation I: Spectrally Accurate Approximation of the Collision Operator, SIAM Journal on Numerical Analysis 37 (2000), no. 4, 1217-1245. 
[17] _ An introduction to Monte Carlo method for the Boltzmann equation, ESAIM: Proc. 10 (2001), 35-75.

[18] C. W. Shu, Essentially non-oscillatory and weighted essentially non-oscillatory schemes for hyperbolic conservation laws, Advanced Numerical Approximation of Nonlinear Hyperbolic Equations (B. Cockburn, C. Johnson, C.W. Shu, and E. Tadmor, eds.), vol. 1697, Springer, 1998, Lecture Notes in Mathematics, pp. 325-432.

[19] C. W. Shu and S. J. Osher, Efficient implementation of essentially non-oscillatory shock capturing schemes II, J. Comput. Phys. 83 (1989), 32-78. 
a)

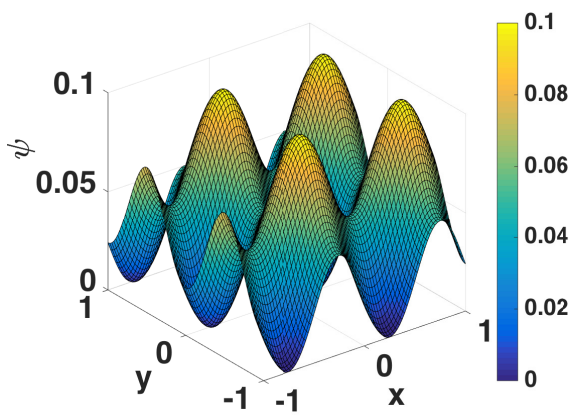

c)

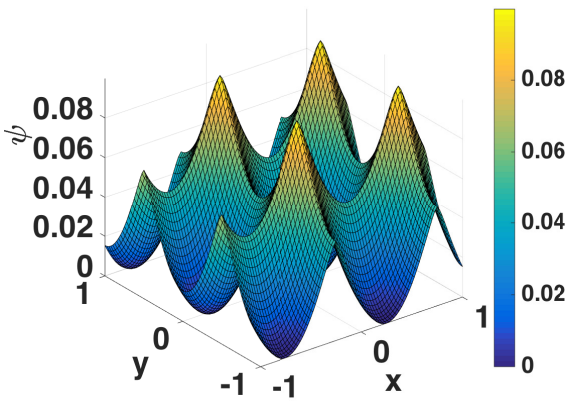

e)

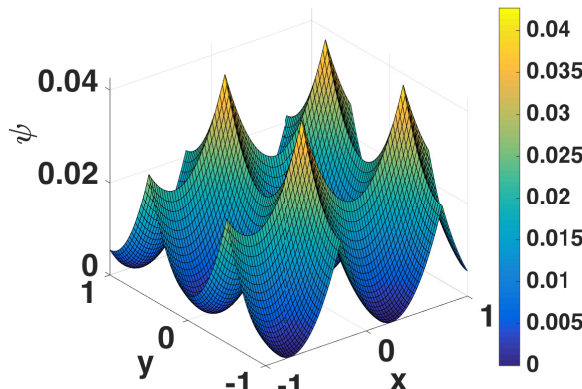

f)

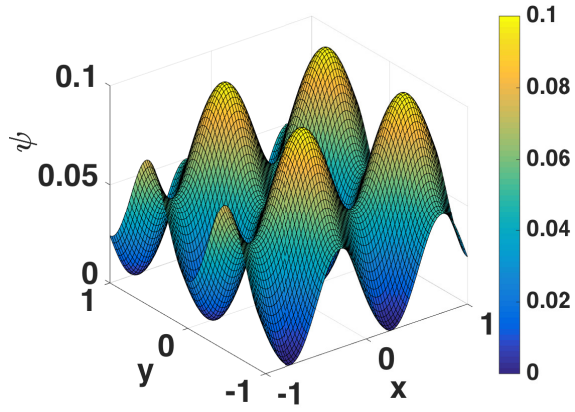

d)
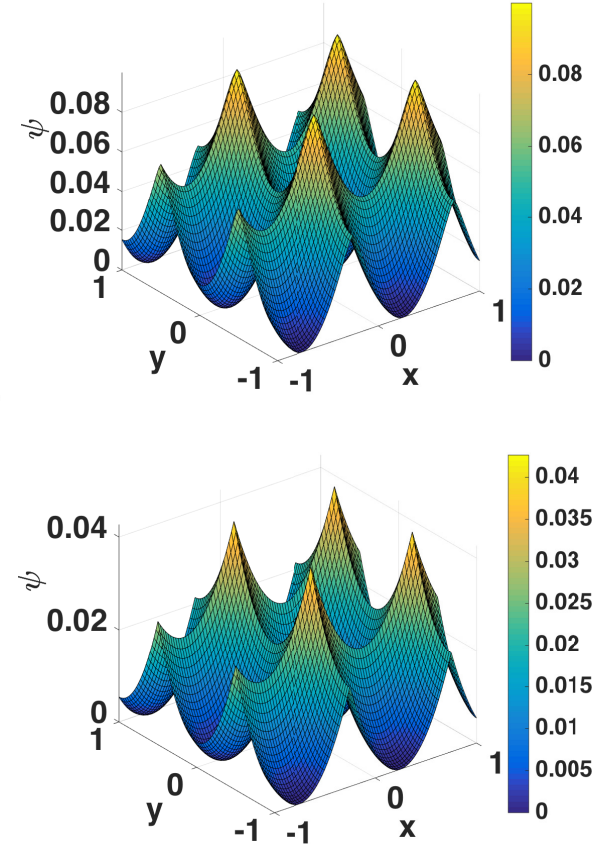

Figure 5: Numerical solutions for Example 2: mesh $81 \times 81$. The solutions $\psi$ are computed by Algorithm 2 with Godunov scheme, where 16 abscissas/weights are used in the Gauss quadrature formula. (a) and (b): at $t=0.1$; (c) and (d): at $t=1$; (e) and (f): at $t=5$. Left: with WENO3-RK3-Godunov ; Right: with WENO5-RK5-Godunov. 


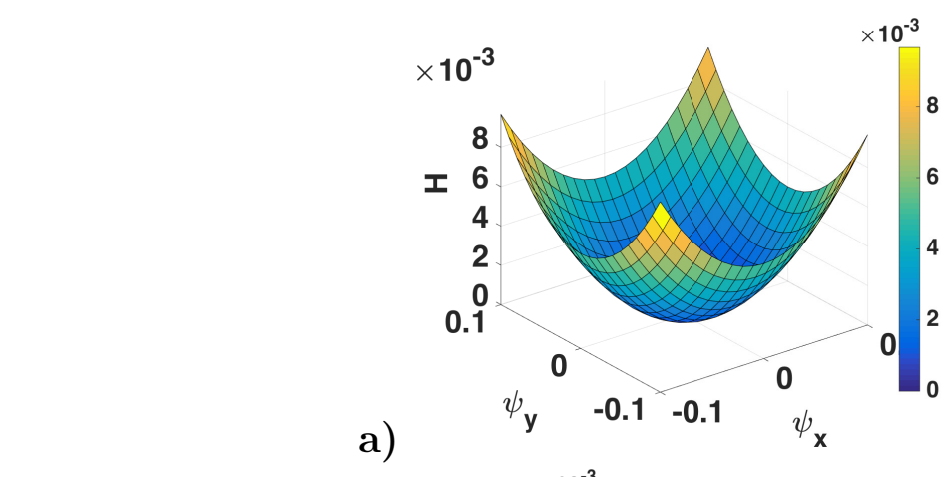

b)

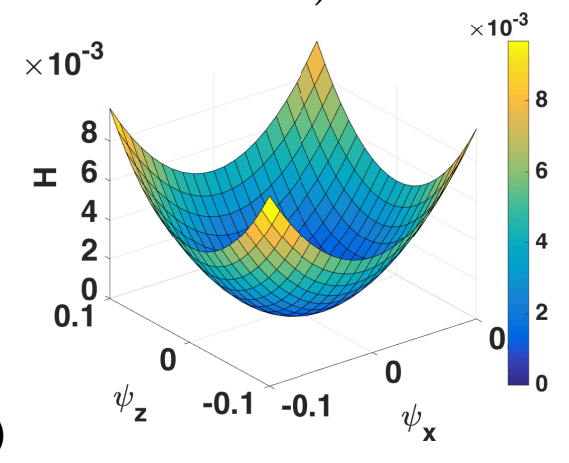

c)

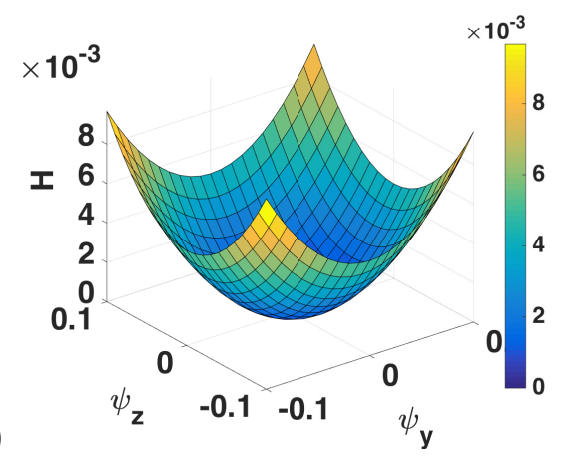

Figure 6: Effective Hamiltonian for Example 3: mesh $21 \times 21 \times 21$ for $\left(\psi_{x}, \psi_{y}, \psi_{z}\right)$. The effective Hamiltonian is computed by Algorithm 1 where 8 abscissas/weights are used in the Gauss quadrature formula. (a) slice at $\psi_{z}=0$; (b) slice at $\psi_{y}=0$; and (c) slice at $\psi_{x}=0$. 
a)

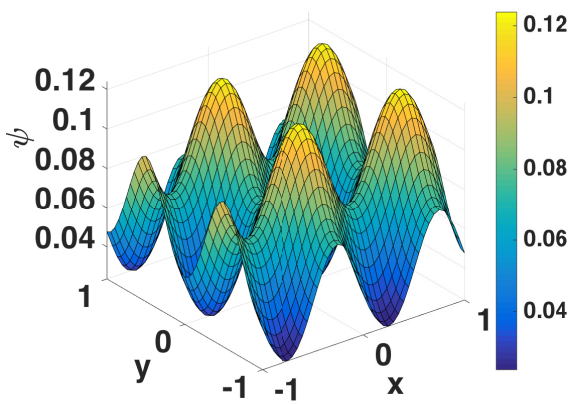

c)

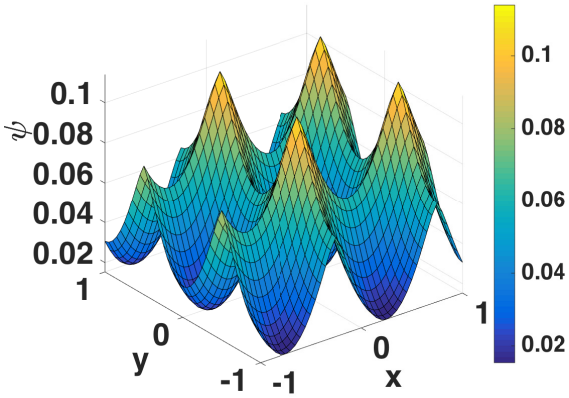

e)

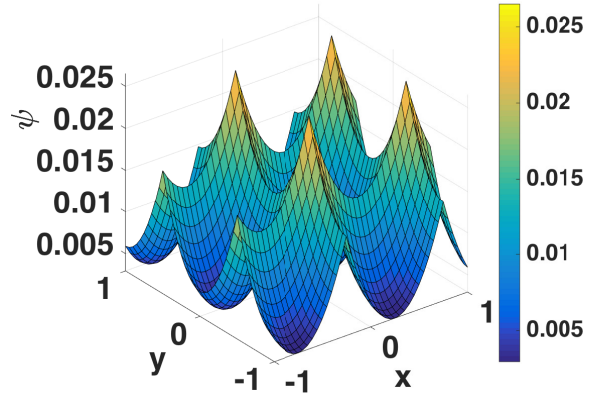

f)

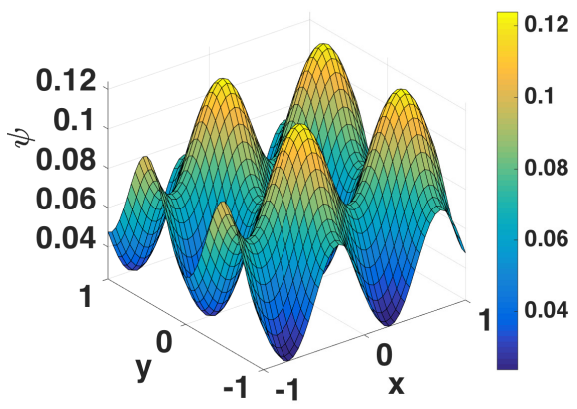

b)

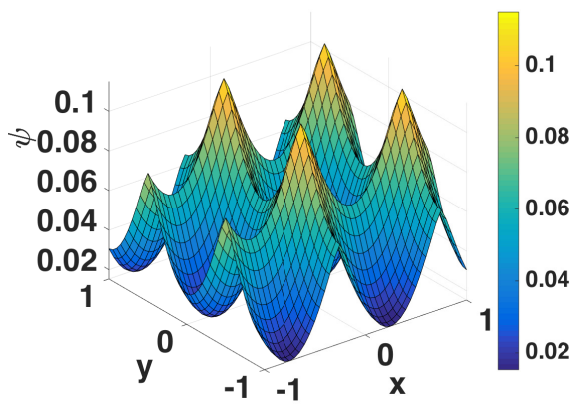

d)

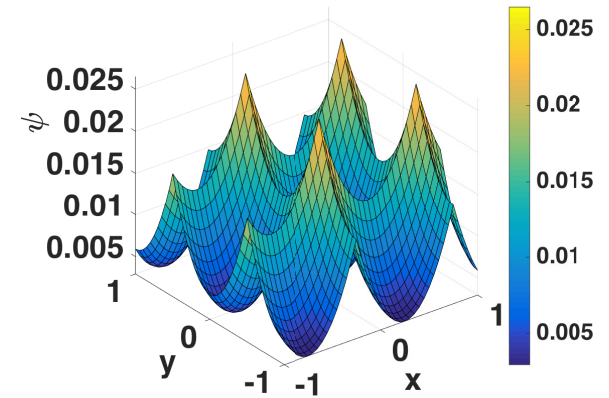

Figure 7: Numerical solutions for Example 3: mesh $41 \times 41 \times 41$. The solutions $\psi$ are computed by Algorithm 2 with Godunov scheme, where 8 abscissas/weights are used in the Gauss quadrature formula. (a) and (b): at $z=0, t=0.1 ;(\mathrm{c})$ and (d): at $z=0, t=1$; (e) and (f): at $z=0, t=10$. Left: with WENO3-RK3-Godunov ; Right: with WENO5-RK5-Godunov. 